\title{
Abundances of PAHs in the ISM: confronting observations with experimental results ${ }^{\star}$
}

\author{
R. Gredel ${ }^{1}$, Y. Carpentier ${ }^{2}$, G. Rouillé2 ${ }^{2}$ M. Steglich ${ }^{2}$, F. Huisken² ${ }^{2}$ and Th. Henning ${ }^{1}$ \\ 1 Max Planck Institute for Astronomy (MPIA), Königstuhl 17, 69117 Heidelberg, Germany \\ e-mail: gredel@mpia.de \\ ${ }^{2}$ Laboratory Astrophysics Group of the Max Planck Institute for Astronomy at the Friedrich Schiller University Jena, \\ Institute of Solid State Physics, Helmholtzweg 3, 07743 Jena, Germany
}

Received 28 January 2011 / Accepted 17 February 2011

ABSTRACT

\begin{abstract}
Context. The identification of the carriers of the diffuse interstellar bands (DIBs) is the longest standing problem in the study of the interstellar medium. Here we present recent UV laboratory spectra of various polycyclic aromatic hydrocarbons (PAHs) and explore the potential of these molecules as carriers of the DIBs. Whereas, in the near IR range, the PAHs exhibit vibrational bands that are not molecule-specific, their electronic transitions occurring in the UV/vis provide characteristic fingerprints. The comparison of laboratory spectra calibrated in intensity with high signal-to-noise observational data in the UV enables us to establish new constraints on PAH abundances.

Aims. From a detailed comparison of the gas-phase and Ne-matrix absorption spectra of anthracene, phenanthrene, pyrene, 2,3-benzofluorene, benzo[ghi]perylene, and hexabenzocoronene with new interstellar spectra, we aim to infer the abundance of these PAHs in the interstellar medium.

Methods. We present spectra of PAHs measured at low temperature in the gas phase and in an Ne matrix, and present methods to derive absolute absorption cross sections for the matrix and gas phase spectra. We have obtained high signal to noise $(S / N>100)$ absorption spectra toward five lines of sight with reddenings of $E_{B-V}=1-1.6$ mag. The spectra cover the 3050-3850 $\AA$ wavelength region where the PAHs studied here show prominent absorption features.

Results. From the observations, we infer upper limits in the fractional abundances of the PAHs studied here. Upper limits in the column densities of anthracene of $0.8-2.8 \times 10^{12} \mathrm{~cm}^{-2}$ and of pyrene and 2,3-benzofluorene ranging from $2-8 \times 10^{12} \mathrm{~cm}^{-2}$ are inferred. Upper limits in the column densities of benzo[ghi]perylene are $0.9-2.4 \times 10^{13}$ and $10^{14} \mathrm{~cm}^{-2}$ for phenanthrene. The measurements indicate fractional abundances of anthracene, pyrene, and 2,3-benzofluorene of a few times $10^{-10}$. Upper limits in the fractional abundance of benzo[ghi]perylene of a few times $10^{-9}$ and of phenanthrene of few times $10^{-8}$ are inferred. Toward CPD $-32^{\circ} 1734$, we found near $3584 \AA$ an absorption line of $\mathrm{OH}^{+}$, which was discovered in the interstellar medium only very recently.

Conclusions. The fractional abundances of PAHs inferred here are up to two orders of magnitude lower than estimated total PAH abundances in the interstellar medium. This indicates that either neutral PAHs are not abundant in translucent molecular clouds or that a PAH population with a wide variety of molecules is present.
\end{abstract}

Key words. ISM: abundances - ISM: molecules - ISM: clouds

\section{Introduction}

The longstanding mystery concerning the nature of the carriers of the diffuse interstellar bands (DIBs) has received considerable and renewed attention in recent years after laboratory spectra of potential candidates have become available. Most scientists in the field support the general idea that the DIBs arise from electronic transitions of gas-phase molecules. Recent examples include the $2_{0}^{2}, 2{ }_{0}^{1} 4_{0}^{1}$, and $2_{0}^{1}$ transitions in the $B^{1} \mathrm{~B}_{1} \leftarrow$ $X^{1} \mathrm{~A}_{1}$ system of $l-\mathrm{C}_{3} \mathrm{H}_{2}$ (Maier et al. 2011), the $(0,0)$ band of the $A^{2} \Pi_{u}-X^{2} \Pi_{g}$ system of the $\mathrm{HC}_{4} \mathrm{H}^{+}$diacetylene cation (Krełowski et al. 2010b), or the anthracene and naphtalene cations (Iglesias-Groth et al. 2008, 2010). Similarly, a recent theoretical work established the excellent agreement between the $0_{0}^{0}$ band of the ${ }^{1} B_{1}-X^{1} A^{\prime}$ electronic system of $\mathrm{CH}_{2} \mathrm{CN}^{-}$with the $8037 \AA$ DIB (Cordiner \& Sarre 2007).

DIBs are generally observed in absorption against the continua of reddened supergiants in the Galaxy and in extragalactic

* Based on observations collected at the European Southern Observatory, Chile (079.C-0597(A)). sources. The appearance of DIBs in translucent molecular clouds characterized by a wide range of physical parameters indicates that DIBs arise from chemically stable carriers. More than 400 DIBs have been identified toward a single galactic line of sight (Hobbs et al. 2009). The individual absorption profiles and line strength ratios of DIBs may vary significantly from one sightline to another (Galazutdinov et al. 2008). While the equivalent widths of several DIBs correlate well with the reddenings $E_{B-V}$ of the background stars, the majority of the DIB carriers appear to be enhanced in the outer regions of translucent clouds (Cami et al. 1997; Sonnentrucker et al. 1997). Observations of DIBs in the Magellanic Clouds (Welty et al. 2006) have shown that some DIBs are significantly weaker than toward Galactic lines of sight with similar reddening, suggesting that the abundance of the responsible carriers scales with the metallicity of the environment. General scaling relations have not been established, however, because the equivalent widths of the family of $\mathrm{C}_{2}$-DIBs, for example, are as strong as in the Galaxy, for lines of sight with comparable reddening (Welty et al. 2006). 
The vibronic progressions established for several DIBs (Duley \& Kuzmin 2010) point toward an origin from a torsional motion of pendent rings, suggesting that the carriers are "floppy" polycyclic aromatic hydrocarbon (PAH) structures, such as 9-phenylanthracene, parasubstituted biphenyls, tetracene derivatives, bianthracene, or tolane, rather than compact molecules. The presence of PAHs in the interstellar medium is now generally established, and there is unequivocal evidence that the unidentified near-infrared emission bands arise from PAHs (Leger \& Puget 1984; Allamandola et al. 1985; Tielens 2008; Bauschlicher et al. 2010). Mixtures of PAHs or nanosized hydrogenated dust particles have been proposed to explain the $2175 \AA$ UV bump seen toward stars reddened by translucent material (Joblin et al. 1992; Mennella et al. 1998; Steglich et al. 2010), and the presence of carbonaceous dust particles in translucent material is well established (Henning \& Salama 1998). The first detection of the $C_{60}$ and $C_{70}$ fullerenes in the young planetary nebula Tc 1 has recently been reported by Cami et al. (2010).

With the present study, we have undertaken a new effort to search for PAHs in translucent molecular clouds. While ionized PAHs exhibit absorption features in the visible and near IR, making them attractive carrier candidates for the DIBs (Salama et al. 1999), the electronic transitions of their neutral counterparts are shifted to the UV. Since neutral PAHs can be prepared more easily with sufficient density in the gas phase and because observational spectra in the UV can be obtained with high $S / N$, we focused our interest on this class of molecules. In this paper, we present results on our search for anthracene, phenanthrene, pyrene, 2,3-benzofluorene, and benzo[ghi]perylene. These molecules were measured in the gas phase with our supersonic jet/CRDS apparatus and show prominent absorption features at wavelengths shorter than $4000 \AA$. We have obtained high signal-to-noise ratio $(S / N>100)$ spectra toward heavily reddened supergiants in the 3050-3850 region using UVES. We developed techniques to calibrate the CRDS spectra against matrix and solution spectra and to derive absolute absorption cross sections for vibronic bands measured in the gas phase. We also searched for hexabenzocoronene, for which we only have $\mathrm{Ne}$ and Ar matrix spectra. We present a method to estimate gas phase transition wavelengths from the matrix spectra. These methods are described in detail in Sect. 2, which also provides the measured gas phase absorption wavelengths and the estimated absorption cross sections of the PAHs studied here. The astronomical spectra, obtained toward earlytype supergiants with reddenings up to $E_{B-V}=1.6 \mathrm{mag}$, are presented in Sect. 3. A detailed comparison with the laboratory results is given in Sect. 4.1, and Sect. 4.2 presents upper limits of PAH column densities toward the lines of sight studied here. Section 4.3 contains an analysis of various diatomic molecular absorption lines which are detected toward the observed stars, and which are used for consistency checks with previous results. The inferred upper limits and the corresponding maximal fractional abundances of anthracene, phenanthrene, pyrene, 2,3-benzofluorene, benzo[ghi]perylene, and hexabenzocoronene are compared to expectations of PAH abundances in translucent material in Sect. 5.2.

\section{Laboratory data}

The confirmation of potential DIB carrier molecules requires laboratory spectra that are obtained under conditions that match the conditions in interstellar clouds as close as possible. Such conditions - collision-free environments and low temperatures prevail in the expansion of supersonic jets of rare gases seeded with the molecule to be studied. In combination with cavity ring-down laser absorption spectroscopy (CRDS) (O'Keefe \& Deacon 1988), low-temperature gas phase absorption spectra are obtained, providing the ultimate data which can be compared with astrophysical observations. For several reasons, however, supersonic jet CRDS spectra are difficult to obtain. It is for instance difficult to transfer complex molecules into the gas phase. In such cases, another low-temperature technique, matrix isolation spectroscopy (MIS) (Bondybey et al. 1996), is performed. In MIS experiments, the sample molecules are dispersed in a transparent matrix of rare gas atoms such as $\mathrm{Ne}$ or Ar kept at cryogenic temperatures of $6.5 \mathrm{~K}$ (Ne-matrix) and $12 \mathrm{~K}$ (Ar-matrix). Compared to laser spectroscopy in supersonic jets, the MIS technique has the advantage that wide ranges of wavelengths are scanned at once and that only small amounts of the sample molecules are required in general. The main disadvantage of MIS experiments is that the molecules are no longer in a collision-free environment. Interactions with the surrounding matrix material induce shifts of the transition energies and a broadening of the absorption profiles. Spectra obtained from MIS experiments are thus of limited use in the astrophysical context. On the other hand, CRDS experiments do not generally allow one to obtain absorption cross sections, as it is difficult to measure the density of the relevant molecules in the supersonic jets. This is more easily achieved for MIS spectra which can be readily compared with solvent spectra, where the concentration of the dissolved molecules can be accurately measured. We have developed methods to derive absolute absorption cross sections for supersonic jet spectra and to determine gas phase absorption wavelengths from MIS spectra, and present them in Sects. 2.1 and 2.2 below.

From the various PAHs studied in our laboratory, we have selected six species revealing prominent absorption features in the 3050-3850 A region, and for which CRDS spectra are available (Rouillé et al. 2004, 2007; Staicu et al. 2004, 2008). In the order of increasing size, these molecules are anthracene, phenanthrene, pyrene, 2,3-benzofluorene, benzo[ghi]perylene, and hexa-peri-hexabenzocoronene (hexabenzocoronene in the following). The structures of the six molecules are illustrated in Fig. 1. Calibrated solution spectra obtained in the nonpolar solvent cyclohexane for anthracene, phenanthrene, pyrene, and benzo[ghi]perylene (Karcher et al. 1985) and in 1,2,4-trichlorobenzene for hexabenzocoronene (Hendel et al. 1986) are used as references for the determination of absolute absorption cross sections as described in the next section. The solution spectrum of 2,3-benzofluorene has been measured in our laboratory, using cyclohexane as solvent. Spectra of anthracene and pyrene have been measured as well and compared with the literature data to validate our experimental setup. The matrix isolation spectra of the six molecules were recorded in $\mathrm{Ne}$ at $6.5 \mathrm{~K}$ with an apparatus described elsewhere (Steglich et al. 2010). For hexabenzocoronene, the calibration procedure has been restricted to an Ne matrix spectrum (Rouillé et al. 2009) since a jet spectrum in the wavelength range of interest is not available.

\subsection{Determination of absolute absorption cross sections for supersonic jet spectra}

In cavity ring-down spectroscopy (CRDS) experiments, the determination of vibrational absorption cross sections is generally 
a)

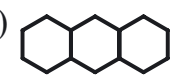

c)

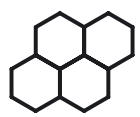

e)

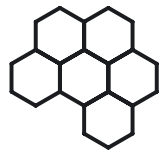

b)<smiles>C1CCC2CCCCC2C1</smiles>

d)
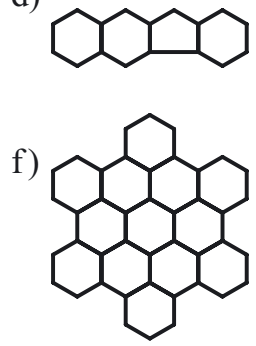

Fig. 1. Schematic carbon structures of the PAHs discussed in this study. a) anthracene, $\mathrm{C}_{14} \mathrm{H}_{10}$; b) phenanthrene, $\mathrm{C}_{14} \mathrm{H}_{10}$; c) pyrene, $\mathrm{C}_{16} \mathrm{H}_{10}$; d) 2,3-benzofluorene, $\mathrm{C}_{17} \mathrm{H}_{12}$; e) benzo[ghi]perylene, $\mathrm{C}_{22} \mathrm{H}_{12}$; f) hexabenzocoronene, $\mathrm{C}_{42} \mathrm{H}_{18}$.

not possible, as the determination of the densities in the supersonic jets remains difficult. In contrast, absolute absorption cross sections are routinely obtained from solvent spectra (Karcher et al. 1985), where the absorption is expressed in terms of a molar extinction coefficient $\epsilon$ (in $1 \mathrm{~mol}^{-1} \mathrm{~cm}^{-1}$ ). Indeed, assuming that absorption is the only significant contribution to the extinction, $\epsilon$ can be converted into an absorption cross section $\sigma$ (in $\mathrm{cm}^{2}$ ) according to

$\sigma=\frac{1000 \ln 10}{N_{\mathrm{A}}} \epsilon=3.8235 \times 10^{-21} \epsilon$

where $N_{\mathrm{A}}$ is the Avogadro constant. By integrating $\sigma$ over a wavelength interval that includes a specific absorption band, one obtains the integrated cross section of the band. It can be used to derive molecular column densities from interstellar spectra using the formalism summarized in Sect. 4.2 (in particular Eq. (5)).

Solvents modify the band intensities of the dissolved molecules and cause shifts of the transition wavelengths and a broadening of the vibronic bands. The effect of the wavelength shifts will be addressed in detail in Sect. 2.2. Due to the broadening, an absorption peak measured in solution may correspond to several vibronic bands. However, if solution spectra are sufficiently well-resolved, they may be used to derive estimates of the vibrational absorption cross section for matrix spectra (Ehrenfreund et al. 1992) or directly for jet spectra (Kokkin et al. 2008). The method is based on the reasonable assumption that the absorption cross section of a molecule integrated over an entire electronic transition is independent of the environment of the molecule, i.e. whether it is vacuum, a rare gas matrix, or a solution. The error that we introduce when we make this assumption is discussed in the next paragraph. For the calibration of our high-resolution gas phase spectra, we have developed a procedure where we use a matrix spectrum as an intermediate step between the solution spectrum and the jet spectrum. We integrate the complete region corresponding to an electronic transition in the solution and matrix spectra and equal them. The same operation is then repeated for the single band of interest in the matrix and jet spectra. The calibration procedure is exemplified in Figs. 2 and 3 for the case of 2,3-benzofluorene. Both figures show the measured absorption profiles of 2,3-benzofluorene in an Ne matrix, in a solution, and in a jet. The different shifts of the band groups seen in Fig. 2 allow us to distinguish between the different electronic transitions of the molecule. The calibration has been applied exclusively to the first electronic transition $\left(S_{1} \leftarrow S_{0}\right)$ of 2,3-benzofluorene. The direct comparison of the

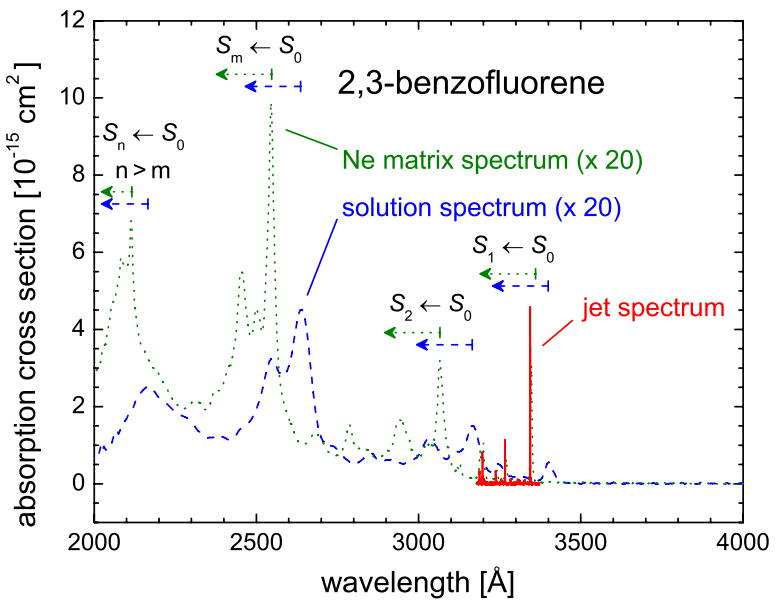

Fig. 2. Absorption spectra of 2,3-benzofluorene measured in a supersonic jet (red full curve), in an Ne matrix at $6.5 \mathrm{~K}$ (green dotted curve), and in cyclohexane at room temperature (blue dashed curve). Note that the matrix and solution spectra were magnified by a factor of 20 . All spectra were measured in our laboratory.

areas of the higher-energy transitions in the solution and matrix spectra is actually complicated by the presence of a continuum in the matrix spectrum attributed to the scattering by the PAH molecules themselves. In Fig. 3, the spectra of the first electronic transition are displayed in wave number, and the matrix and solution spectra are shifted to the blue to account for environment effects ( 34 and $491 \mathrm{~cm}^{-1}$, respectively). To calibrate the matrix spectrum against the solution spectrum, the integration has been performed from 29500 to $31500 \mathrm{~cm}^{-1}$ (see Fig. 3). The area of the gas phase origin band at $29894 \mathrm{~cm}^{-1}$ has then been equaled to the corresponding band at $3348 \AA$ in the matrix spectrum while the contribution of the smaller band at $29872 \mathrm{~cm}^{-1}$ has been subtracted.

In order to estimate the error that we introduce with the assumption that the integrated cross section is not affected by the environment of the molecule, we have to consider the electronic interaction between the solute molecule and the neighboring solvent molecules. The variations of the band intensities caused by this interaction are described by various models (Chako 1934; Linder \& Abdulnur 1971). In the first approximation, the Lorentz-Lorenz force which arises from the polarization of the surrounding solvent molecules can be described in terms of the Chako factor $9 n /\left(n^{2}+2\right)^{2}$, where $n$ is the refractive index of the solvent. Multiplying the oscillator strength of a band measured in solution by the Chako factor, the corresponding oscillator strength in the gas phase is obtained. These models have been successfully applied to $\mathrm{C}_{60}$ by Smith (1996). For the wavelength range and the experiments discussed here, where cyclohexane was used as solvent, the Chako factor is 0.78 . In view of the fact that the Chako factor is still an approximation and that the proper ratio of the oscillator strengths in the gas phase and in a solution depends on the exact nature of both the solvent and solute molecules, we chose not to apply any correction factor to our cross section values. Taking into account the other sources of errors including the experimental and data processing uncertainties, we estimate that the oscillator strengths are accurate within $\pm 50 \%$.

The results of the calibration procedure are summarized in Table 1. The wavelength positions and widths are from our CRDS experiments, except for hexabenzocoronene, for which the wavelength position in the gas phase was extrapolated from 
Table 1. Spectral properties (attribution, position, width, integrated cross section, and oscillator strength) of the PAH bands considered for comparison with astrophysical data in this article.

\begin{tabular}{lcccccc}
\hline \hline Molecule & Electronic transition & $\begin{array}{c}\lambda_{\text {air }}{ }^{a} \\
(\AA)\end{array}$ & $\begin{array}{c}F W H M \\
(\AA)\end{array}$ & $\begin{array}{c}\int \sigma \mathrm{d} \lambda \\
\left(10^{-16} \mathrm{~cm}^{2} \AA\right)\end{array}$ & $\begin{array}{c}N_{\max ^{b}} \\
\left(\mathrm{~cm}^{-2}\right)\end{array}$ \\
\hline $\begin{array}{l}\text { anthracene } \\
\text { phenanthrene }\end{array}$ & $S_{1} \leftarrow S_{0}$ & $3610.74^{c}$ & 0.34 & 19 & 0.016 & $5.3 \times 10^{11}$ \\
pyrene & $S_{1} \leftarrow S_{0}$ & $3409.21^{d}$ & 0.20 & 0.16 & 0.00016 & $6.2 \times 10^{13}$ \\
& $S_{2} \leftarrow S_{0}$ & $3208.22^{e}$ & 5.5 & 89 & 0.097 & $1.1 \times 10^{11}$ \\
2,3-benzofluorene & & $3166.26^{e}$ & 4.7 & 30 & 0.033 & $3.4 \times 10^{11}$ \\
& $S_{1} \leftarrow S_{0}$ & $3344.16^{f}$ & 0.18 & 8.4 & 0.0085 & $1.2 \times 10^{12}$ \\
benzo[ghi]perylene & & $3266.63^{f}$ & 0.22 & 2.4 & 0.0025 & $4.2 \times 10^{12}$ \\
& $S_{2} \leftarrow S_{0}$ & $3512.15^{g}$ & 8.2 & 9.9 & 0.009 & $1 \times 10^{12}$ \\
hexabenzocoronene & & $3501.76^{g}$ & 5.3 & 5.7 & 0.005 & $1.8 \times 10^{12}$ \\
\hline
\end{tabular}

Notes. ${ }^{(a)}$ Band positions are given for air under standard conditions (see text); ${ }^{(b)}$ an upper limit of the column density has been calculated assuming a detection limit with an equivalent width of $1 \mathrm{mÅ} ;{ }^{(c)}$ Staicu et al. (2004); ${ }^{\left({ }^{(d)}\right.}$ Rouillé et al. (2010); ${ }^{(e)}$ Rouillé et al. (2004); ${ }^{(f)}$ Staicu et al. (2008); (g) Rouillé et al. (2007); ${ }^{(h)}$ extrapolated from Ne and Ar matrix measurements (Rouillé et al. 2009b); ${ }^{(i)}$ calculated for Ne matrix spectra.

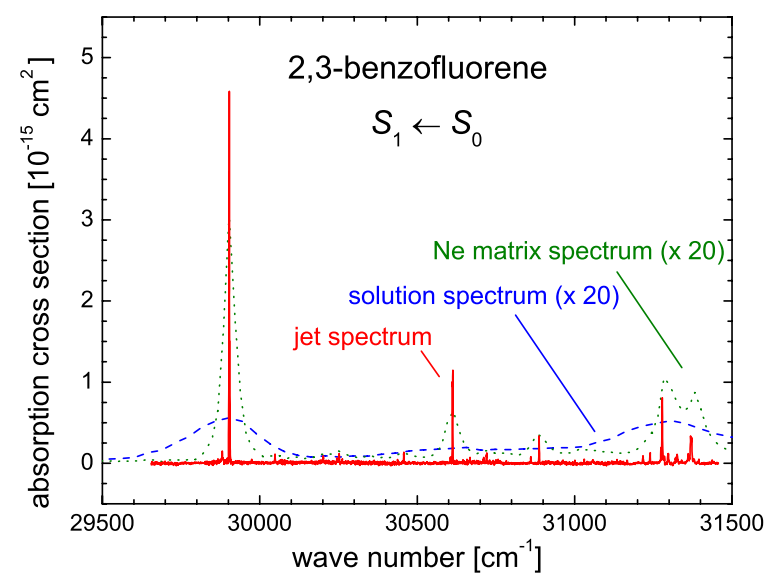

Fig. 3. $S_{1} \leftarrow S_{0}$ absorption spectra of 2,3-benzofluorene under the same experimental conditions as in Fig. 2. The solution and matrix spectra have been shifted to higher energy by $491 \mathrm{~cm}^{-1}$ and $34 \mathrm{~cm}^{-1}$, respectively.

matrix measurements (cf. Sect. 2.2). Although the band positions were originally determined for vacuum, we converted them to air because high-resolution astronomical spectra are traditionally calibrated against $\mathrm{Th} / \mathrm{Ar}$ lines whose wavelengths are given for air. The conversion was carried out according to $\lambda_{\text {air }}=\lambda_{\text {vac }} / n$ with $n$ being the index of refraction of air under standard conditions at $\lambda_{\text {vac }}$, as computed with Eq. (A1) of Livengood et al. (1999). For anthracene and phenanthrene, the results are given for the origin of their respective $S_{1} \leftarrow S_{0}$ transitions. For pyrene, the spectrum of the $S_{2} \leftarrow S_{0}$ transition is composed of band clusters due to the vibronic interaction between the $S_{2}$ and $S_{1}$ states. We give in Table 1 the central wavelengths and the total FWHMs of the two band groups at $3166 \AA$ and $3208 \AA$ (Rouillé et al. 2004). For 2,3-benzofluorene, the origin and a strong vibronic band of the $S_{1} \leftarrow S_{0}$ transition are listed. For benzo[ghi]perylene, the spectral region around $3500 \AA$ shows two broad bands attributed to vibrationally excited bands of the $S_{2} \leftarrow S_{0}$ transition. We also list the oscillator strength $f$ obtained in the approximation of weak bands (Mulliken 1939) from

$f=\frac{4 \epsilon_{0} m c^{2}}{e^{2} \lambda^{2}} \int \sigma(\lambda) \mathrm{d} \lambda$,
Table 2. Comparison of the energies and oscillator strengths for the first electronic transition of 2,3-benzofluorene in calculated and experimental spectra.

\begin{tabular}{lccc}
\hline \hline Chemistry model & $\begin{array}{c}\text { Energy } \\
\mathrm{cm}^{-1}\end{array}$ & $f$ & Ref. \\
\hline ZINDO & 30095 & 0.0116 & $a$ \\
CIS(D)/6-31G(d) & 34923 & 0.0234 & $a$ \\
TD-DFT-B3LYP/TZ & 30431 & 0.038 & $a$ \\
Ar matrix & 29647 & 0.024 & $b$ \\
supersonic jet & 29894.3 & 0.03 & ${ }^{c}$ \\
\hline
\end{tabular}

Notes. ${ }^{(a)}$ Staicu et al. (2008); ${ }^{(b)}$ Banisaukas et al. (2004); ${ }^{(c)}$ Staicu et al. (2008) and this work.

where $\lambda$ is the central wavelength of the band and $\epsilon_{0}, c, m$, and $e$ are the vacuum permittivity, the speed of light in vacuum, the mass, and the charge of the electron, respectively. It is interesting to note that, in some cases, the absorption cross sections and oscillator strengths vary significantly for the different molecules studied especially if we consider the transition with the lowest energy $\left(S_{1} \leftarrow S_{0}\right)$. Thus, we found that the oscillator strengths of this transition differ by two orders of magnitude if we compare the two molecules anthracene and phenanthrene, which are both composed of three aromatic cycles (see Fig. 1). This shows that the change of the electronic structure during the transition, which determines the strength of the transition, does not obey simple empirical rules. In fact, laboratory experiments seem necessary to determine this important property.

In the case of 2,3-benzofluorene, an oscillator strength of 0.0085 is derived for the origin band of the $S_{1} \leftarrow S_{0}$ transition (see Table 1). The summation over the complete electronic transition (i.e. the origin band and the associated vibronic bands) yields an integrated oscillator strength of 0.03 . This value is compared in Table 2 with the results of theoretical calculations (Staicu et al. 2008) and a laboratory measurement (Banisaukas et al. 2004). Theoretical transition energies and oscillator strengths were obtained by applying different quantum chemistry models. In Table 2, we refer to results obtained with a semiempirical model, ZINDO, an ab initio method, CIS(D)/6-31G(d), and a model based on the time-dependent density functional theory, TD-DFT-B3LYP/TZ. For details on 
the computational methods, see Staicu et al. (2008) and references therein. As far as the oscillator strength is concerned, the largest deviation from experimental data is found for the ZINDO calculation although it yields the best result for the transition energy. The oscillator strengths of the other two calculations come close to the experimental values. The satisfactory agreement of our oscillator strength with ab initio calculations and the result derived from Ar matrix data gives us confidence that our method to determine absolute absorption cross sections leads to reasonable results.

For the other PAHs discussed in this paper, absorption cross sections of calculated spectra are available in a database (Malloci et al. 2004, 2007). Unfortunately, the theoretical spectra were convoluted with a too broad line shape function making a comparison with our experimental data very difficult or even impossible.

At this point, a general statement about computed electronic transition energies seems appropriate. As can be inferred from the data of Table 2, the theoretical values may strongly differ from the experimental results. The current quantum chemistry models and calculation techniques allow one to predict electronic transition energies with an accuracy of the order of $0.2 \mathrm{eV}$ at best. The accuracy of semiempirical calculations, even if the models are well parametrized, suffers from their simplicity. On the other hand, the ab initio approach is the most complete. In order to be applied to large molecules, however, approximations have to be made, affecting the accuracy of the results. Finally, calculations based on the density functional theory currently miss functionals adapted to PAH molecules. For our example molecule, 2,3-benzofluorene, the best result is obtained with the semiempirical ZINDO method. However, the deviation from the experimental value is with more than $20 \AA$ still quite significant. In comparison, the extrapolation of matrix data, an experimental method which will be described in the next section, yields a frequency position as close as $5 \AA$ to the gas phase position. In conclusion, we can say that the uncertainty of quantum chemical calculations is still too large for a meaningful comparison with high-precision astronomical observations.

\subsection{Determination of gas phase absorption wavelengths from MIS spectra}

PAH absorption wavelengths obtained from MIS experiments or from solutions suffer from a broadening and a shift of the absorption features, compared to the absorption profiles obtained in an environment free of interactions. It is nevertheless possible to exploit MIS results to predict band positions in the gas phase if they are not available, as in the case of the $\beta$-band of hexabenzocoronene (Rouillé et al. 2009). For PAH molecules in rare gas matrices, the dominating mechanism in the interaction between the molecules and the rare gas atoms is the dispersion effect. The effect arises from the interaction of polarizable species and is proportional to the product of the mean static dipole polarizabilities (London 1937). As the dispersion effect depends on the polarizability of the matrix material, a given transition shows different energy shifts in different matrices, and an extrapolation to a matrix with zero polarizability allows one to estimate the band position in the gas phase. The models of Longuet-Higgins \& Pople (1957) and Shalev et al. (1991a,b, 1992) have been applied to evaluate the transition energy shifts for PAH molecules surrounded by rare gas atoms (Biktchantaev et al. 2002; Shalev et al. 1991a,b, 1992). These models take into account the arrangement of the rare gas atoms around the molecules. As this information cannot be obtained by straightforward methods, we assume that the dispersion effect does not vary significantly when we consider the different geometries encountered for PAH molecules in Ne and Ar matrices. The transition frequency $\tilde{v}_{\text {gas phase }}$ of a band is then obtained as

$\tilde{v}_{\text {gas phase }}=\tilde{v}_{\text {Ne matrix }}+\frac{1}{R_{\alpha}-1}\left(\tilde{v}_{\text {Ne matrix }}-\tilde{v}_{\text {Ar matrix }}\right)$,

where $R_{\alpha}=4.13$ is the ratio of the polarizabilities of $\mathrm{Ar}$ and $\mathrm{Ne}$ (Radzig \& Smirnov 1985).

We apply this method to the complete electronic spectrum of hexabenzocoronene that we have recently measured in an Ne matrix (Rouillé et al. 2009). The full spectrum reveals strong absorption bands in the 3000-4000 $\AA$ spectral region. A well defined feature of the $\alpha$-band was found at $4383 \pm 5$ and $4344 \pm 5 \AA$ in $\mathrm{Ar}$ and $\mathrm{Ne}$, respectively. The linear extrapolation based on the ratio of the rare gas polarizabilities leads to a wavelength of $4332 \pm 9 \AA$. This feature was measured in a cold molecular beam (Kokkin et al. 2008) at a vacuum wavelength of 4335.2 $\AA$, which corresponds to $4334.0 \AA$ in air, a value in close agreement with our prediction. The significantly stronger $\beta$ band of hexabenzocoronene occurs near $3344 \AA$ (Rouillé et al. 2009).

Unlike the $\alpha$-band, the $\beta$-band shows features that are unresolved in the matrix spectra. They correspond to numerous bands that arise through the interaction of the $S_{\beta}$ electronic state with the vibrational manifold of $S_{2}$ (Rouillé et al. 2009). As a consequence, each peak of the $\beta$-band in the matrix spectra is expected to be resolved in the gas phase into a group of bands. For example, such groups are found in the spectrum of the $S_{2} \leftarrow S_{0}$ transition of pyrene (cf. Sect. 4.1). In the case of pyrene, the extrapolation from peaks measured by us at $3302 \pm 4$ and $3234 \pm 5 \AA$ in Ar and Ne matrices, respectively, gives an expected position of $3213 \pm 8 \AA$ of this band in the gas phase. The strongest peak of this group has been measured at $3208.2 \AA$ or $31160.9 \pm 0.5 \mathrm{~cm}^{-1}$ in the gas phase (Rouillé et al. 2004), in good agreement with the result of the extrapolation. Considering now the matrix spectra of hexabenzocoronene, the relative intensities of the peaks in the $\beta$-band vary depending on the matrix material. We assume that the strongest peak in the Ne matrix spectrum corresponds in the gas phase to the group of bands that includes the strongest one. The peak lies at $3420 \pm 4$ and $3362 \pm 5 \AA$ in Ar and Ne (Rouillé et al. 2009), respectively. Our simple model leads to an estimated wavelength of $3344 \pm 8 \AA$ of this band in the gas phase.

\section{Observations and data reduction}

We have obtained high-resolution spectra toward the heavily reddened supergiants HD 169454 (B1.5Ia, $E_{B-V}=1.13 \mathrm{mag}$ ), $\mathrm{BD}-14^{\circ} 5037$ (B1.5Ia, $E_{B-V}=1.56 \mathrm{mag}$ ), $\mathrm{CPD}-32^{\circ} 1734$ (B1.5Ia, $E_{B-V}=1.22 \mathrm{mag}$ ), $\mathrm{CPD}-33^{\circ} 1768$ (B2.5I, $E_{B-V}=$ $1.46 \mathrm{mag}$ ), and HD 183143 (B7Iae, $E_{B-V}=1.27 \mathrm{mag}$ ), using the UVES spectrograph at the VLT under programme 079.C-0597(A). Total integration times were $14 \times 300 \mathrm{~s}$ for $\mathrm{CPD}-32^{\circ} 1734,11 \times 500 \mathrm{~s}$ for CPD $-33^{\circ} 1768,16 \times 250 \mathrm{~s}$ for $\mathrm{BD}-14^{\circ} 5037,20 \times 50 \mathrm{~s}$ for HD 169454 , and $6 \times 50 \mathrm{~s}$ for HD 183143. The spectra are reduced within the ESO MIDAS package following standard procedures and are rebinned to a heliocentric wavelength scale.

We also use the archival UVES spectra obtained under the UVES Paranal Observatory Project "A Library of High-Resolution Spectra of Stars across the HertzsprungRussell Diagram" (Bagnulo et al. 2003) produced under ESO 
Director Discretionary Time (DDT) program 266.D-5655(A). The database of Bagnulo et al. (2003) contains a large number of field stars with spectral types ranging from $\mathrm{O}$ to $\mathrm{M}$ and luminosity classes ranging from main sequence stars to supergiants. Here we select a few of the more reddened luminous $\mathrm{O}-$ and B-type stars with reddenings up to $E_{B-V}=$ $1 \mathrm{mag}, \mathrm{HD} 97253$ (O5.5III, $E_{B-V}=0.50 \mathrm{mag}$ ), HD 148937 (O6.5, $\left.E_{B-V}=0.67 \mathrm{mag}\right), \mathrm{HD} 96917$ (O8.5Ib, $E_{B-V}=$ $0.37 \mathrm{mag}$ ), HD 76341 (O9Ib, $E_{B-V}=0.46 \mathrm{mag}$ ), HD 152003 (O9.7Iab, $E_{B-V}=0.64 \mathrm{mag}$ ), and toward the reddened B-type supergiants HD 112272 (B0.5Ia, $E_{B-V}=0.99 \mathrm{mag}$ ), HD 115363 (B1Ia, $E_{B-V}=0.82 \mathrm{mag}$ ), HD 152235 (B1 Ia, $E_{B-V}=0.73 \mathrm{mag}$ ), and HD 148379 (B1.5Ia, $E_{B-V}=0.71 \mathrm{mag}$ ). Values of $E_{B-V}$ are from Kaźmierczak et al. (2010). The archival spectrum of HD 169454 is used for consistency checks.

The archive contains several stars with low reddening, which we use to identify stellar absorption lines. We select the spectra toward HD 29138 (B1 Iab), HD 58978 (B1 II), and Rigel (B8 Iab), which are characterized by very faint or absent interstellar molecular absorption lines of $\mathrm{CH} 4300 \AA, \mathrm{CH}^{+} 4232 \AA$, and CN $3875 \AA$, and faint or absent Ti I $3229 \AA$ and Na I $3302 \AA$ absorption lines. We use the models of Gummersbach \& Kauffer (1993) to assess the stellar continua of our programme stars. These models are based on the LTE static plane-parallel lineblanketed ATLAS9 model atmosphere of Kurucz (1991) and were developed for main-sequence stars with spectral types ranging from $\mathrm{O} 9 \mathrm{~V}$ to $\mathrm{B} 5 \mathrm{~V}$. The stars in our sample are supergiants in general, some of them with strong stellar winds. Stellar parameters such as gravity, rotation, and metallicity which affect the width and the depth of the photospheric absorption lines may thus deviate significantly from those adopted in the models used here. Because of this, the models of Gummersbach \& Kauffer (1993) may not reproduce the stellar spectra presented here in all details, yet the models are still extremely useful to distinguish among stellar and potential interstellar absorption features in our UVES spectra.

\section{Results}

\subsection{Comparison of astrophysical and laboratory spectra}

In the standard reduction scheme adopted here (cf. Sect. 3), the spectra obtained with UVES are rebinned to a heliocentric wavelength scale. In order to compare the astronomical spectra with our laboratory spectra, we proceed as follows. The astronomical spectra contain a number of interstellar absorption lines from diatomic molecules (cf. Sect. 4.3 and Table 4) which we use to infer average heliocentric velocities $V_{\text {ave }}$ of $-9.8 \mathrm{~km} \mathrm{~s}^{-1}$, $-7.9 \mathrm{~km} \mathrm{~s}^{-1},+36.8 \mathrm{~km} \mathrm{~s}^{-1}$, and $+35.9 \mathrm{~km} \mathrm{~s}^{-1}$ of the molecular material toward HD 169454, BD - $14^{\circ} 5037$, CPD $-33^{\circ} 1768$, and CPD $-32^{\circ} 1734$, respectively. We now shift the stellar spectra by the respective values of $-V_{\text {ave }}$ and plot the modified spectra, together with the laboratory absorption profiles of the PAHs studied here, in Figs. 4-11. Assuming that the sought PAH absorbers reside in the molecular material traced by the diatomic molecules detected here (cf. Table 4), corresponding interstellar absorption features must agree in position and in spectral profile with the laboratory profiles plotted in Figs. 4-11. Note that a velocity dispersion of up to $4 \mathrm{~km} \mathrm{~s}^{-1}$ among the various interstellar absorbers toward a given line of sight would translate into a wavelength spread of about one resolution element only, which is negligible.

Figure 4 presents the comparison of the origin band of the $S_{1} \leftarrow S_{0}$ transition of anthracene, which is represented by the

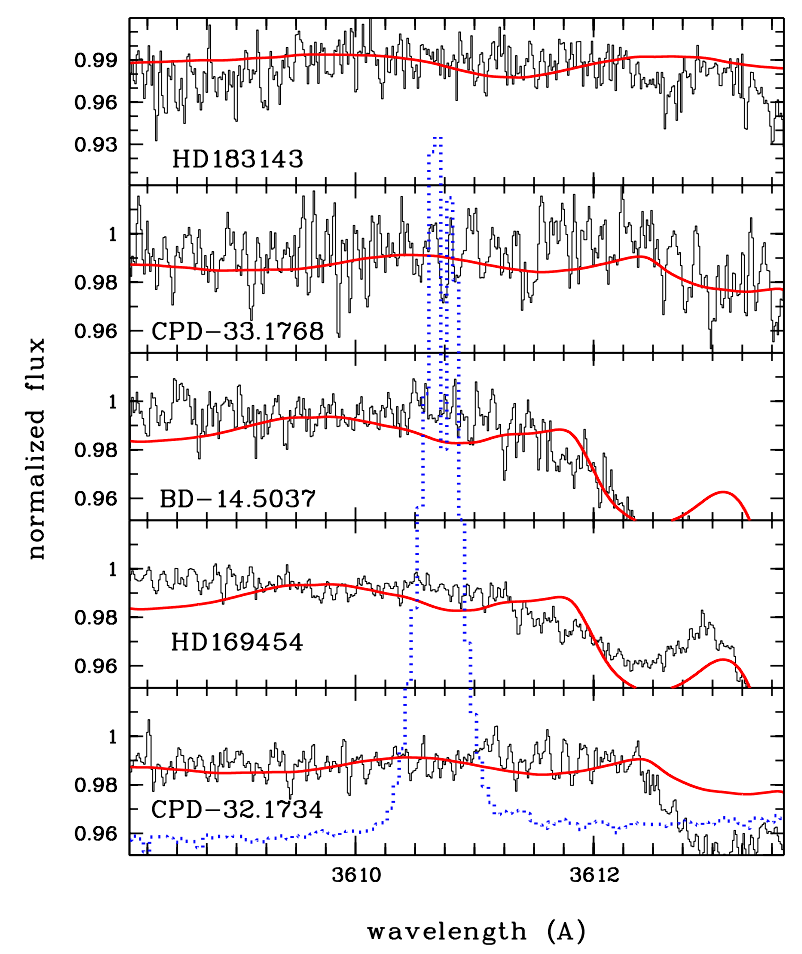

Fig. 4. Comparison between the $S_{1} \leftarrow S_{0}$ origin band of anthracene measured in our laboratory (plotted as dotted blue curve), Staicu et al. (2004) and the UVES spectra obtained toward our programme stars. Red continuous lines represent synthetic stellar spectra obtained from the models of Gummersbach \& Kauffer (1993). All stellar spectra have been rebinned to a wavelength frame where the detected interstellar absorption lines occur at an average radial velocity of $0 \mathrm{~km} \mathrm{~s}^{-1}$, compared with the respective rest wavelengths in air.

dotted blue curve (Staicu et al. 2004), with the UVES spectra of our five programme stars. Red continuous lines represent synthetic stellar profiles obtained from the models of Gummersbach \& Kauffer (1993). Toward HD 169454 and BD -145037, the expected absorption of anthracene agrees in wavelength with a faint photospheric Fe III (3611.7 $\AA$ ) line. Stronger stellar absorption lines at $3612.4 \AA$ and $3613.6 \AA$ correspond to $\mathrm{Al}$ III and $\mathrm{He}$ I, respectively. Toward the two B1.5Ia supergiants HD 169454 and BD $-14^{\circ} 5037$, the stellar continuum near the expected absorption of anthracene is not very well reproduced by the models of Gummersbach \& Kauffer (1993), yet the discrepancies are assigned to effects of stellar parameters such as gravity and metallicity. It can thus be concluded that the spectra shown in Fig. 4 do not reveal signatures of interstellar anthracene absorptions at $3610.74 \AA$ A. The observations may nevertheless be used to infer upper limits in the column density and in the fractional abundance of anthracene (cf. Sect. 4.2) toward our lines of sight.

The laboratory spectrum of the $S_{1} \leftarrow S_{0}$ origin band of phenanthrene at $3409.21 \AA$ is given in Fig. 5 together with the five UVES spectra. The transition is significantly weaker than the $S_{2} \leftarrow S_{0}$ origin band, yet the latter occurs at $2826.42 \AA$ which is not accessible from the ground. The corresponding spectral region toward the B7Ia supergiant HD 183143 shown in Fig. 5 is dominated by stellar Ni II (3407.3 $\AA$ ) and Cr II (3408.8 $)$ ) absorption lines which are well reproduced by the stellar models. The hotter atmospheres of HD 169454 and BD -14 5037 contain faint absorptions aris-

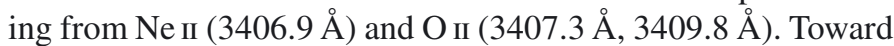
BD $-14^{\circ} 5037$, an absorption feature occurs near $3408 \AA$ which 


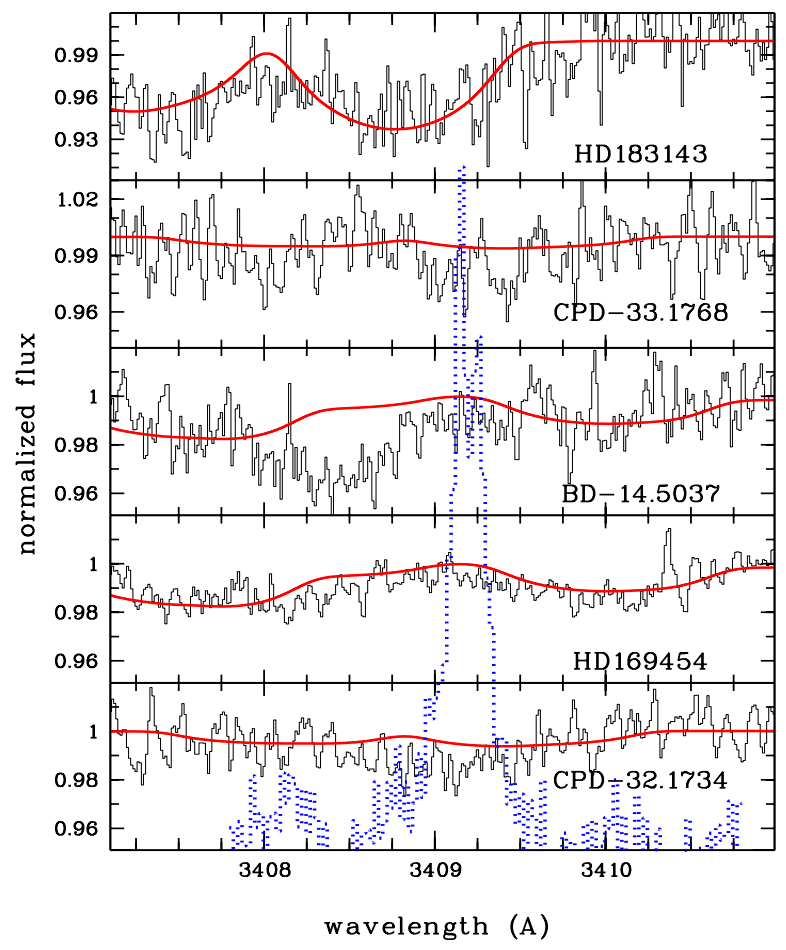

Fig. 5. Comparison of the weak $S_{1} \leftarrow S_{0}$ transition of phenanthrene (dotted blue curve) with UVES spectra of our programme stars. See Fig. 4 for further details.

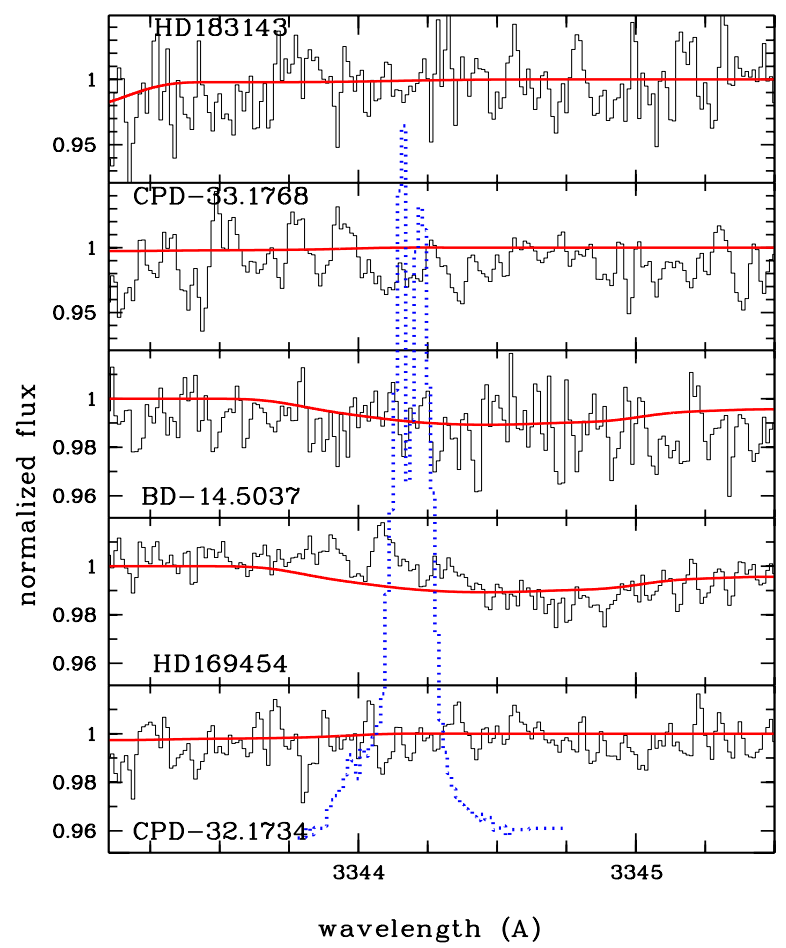

Fig. 6. Comparison of the origin band of the $S_{1} \leftarrow S_{0}$ transition of 2,3-benzofluorene (dotted blue curve) with the UVES spectra of our programme stars. See Fig. 4 for further details.

is not reproduced by the stellar models. The stellar continuum of the unreddened star HD 148379, which is a B1.5 Ia supergiant as well, is featureless in the spectral region shown in Fig. 5. It is thus tempting to assign the absorption feature near $\lambda_{\text {air }}=3408.3 \AA$ toward $\mathrm{BD}-14^{\circ} 5037$ to a new diffuse

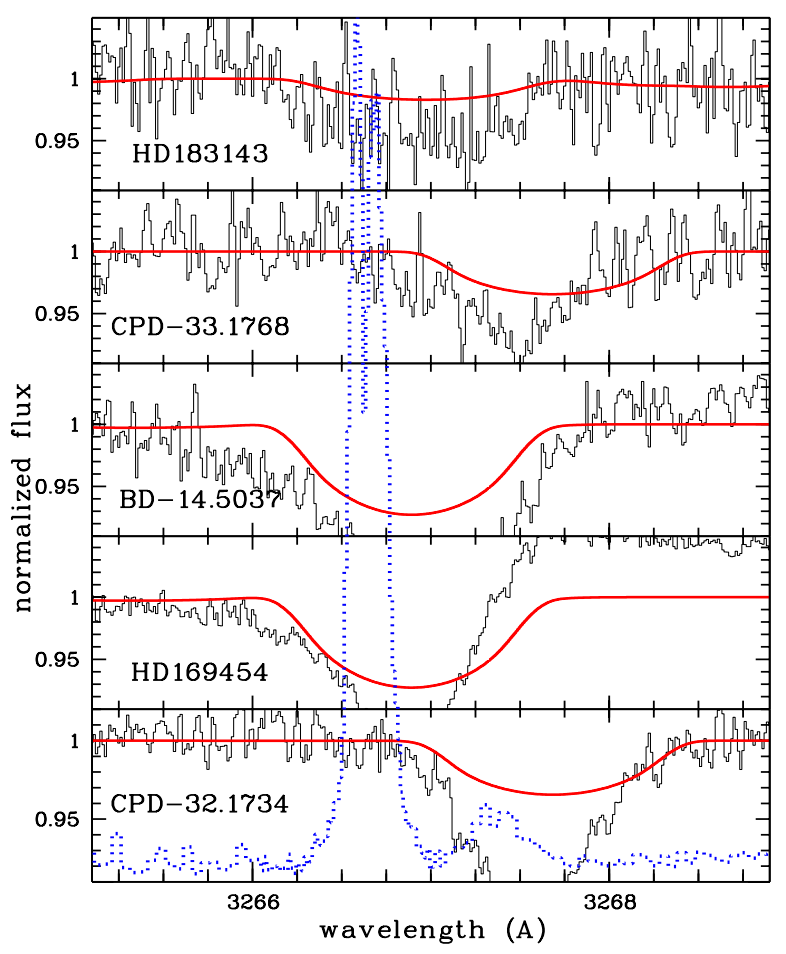

Fig. 7. Comparison of laboratory data of 2,3-benzofluorene (dotted blue curve) with UVES spectra of our programme stars. The dotted blue curve represents an electronic transition involving the additional excitation of a vibration with an energy of $700 \mathrm{~cm}^{-1}$ (Staicu et al. 2008). See Fig. 4 for further details.

interstellar band, yet its origin from phenanthrene is rejected. The absorption feature is significantly broader than the phenanthrene band and it would be blue-shifted by some $100 \mathrm{~km} \mathrm{~s}^{-1}$ if due to phenanthrene. BD $-14^{\circ} 5037$ and HD 169454 are members of the Sct OB3 association where high-velocity gas has been detected (Federman \& Lambert 1992). The high-velocity gas, redshifted by some $100 \mathrm{~km} \mathrm{~s}^{-1}$, has been detected toward HD 169454 and a few other stars in the Sct OB3 association, but not toward $\mathrm{BD} 14^{\circ} 5037$. Its origin has been assigned to an expanding filamentary shell, probably due to a $\mathrm{SN}$ explosion in Sct OB3.

Figures 6 and 7 present the comparison of the laboratory spectra of 2,3-benzofluorene with our UVES spectra. Figure 6 displays the vibrationless origin band of the $S_{1} \leftarrow S_{0}$ transition of 2,3-benzofluorene at $3344.16 \AA$. The hotter stars such as HD 169454 show faint stellar absorption lines arising from Ne II (3344.4 $\AA$ and $3345.5 \AA$ ) and Ar III (3344.7 $\AA$ ) which merge into a shallow and unresolved absorption feature. The relatively featureless stellar continua of our programme stars in this wavelength region favor the search of the relatively narrow $S_{1} \leftarrow S_{0}$ transition of 2,3-benzofluorene, yet none of the spectra show a corresponding absorption feature near $3344.16 \AA$. Figure 7 shows a weaker band of 2,3-benzofluorene located at $3266.63 \AA$. That band involves an additional vibrational excitation. A strong stellar Fe III absorption (3266.9 $\AA$ ) is present in all spectra, and it occurs at the expected position of the 2,3benzofluorene absorption. The Fe III line toward HD 169454 exhibits a strong P Cygni profile. The adopted models do not reproduce the depth nor the profiles of the Fe III line toward CPD $-32^{\circ} 1734$, HD 169454, and BD $-14^{\circ} 5037$. It can thus not be ruled out that additional absorption due to 2,3-benzofluorene 


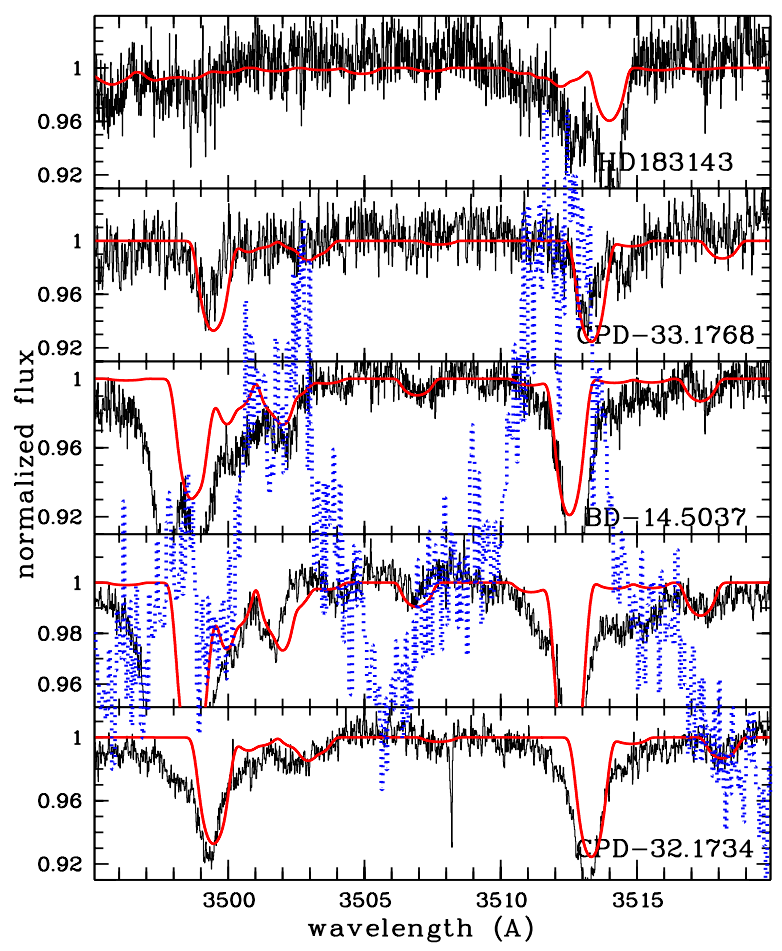

Fig. 8. Comparison of laboratory data of benzo[ghi]perylene (dotted blue curve) with UVES spectra of our programme stars. The laboratory spectrum shows two vibrational bands in the $S_{2} \leftarrow S_{0}$ transition (Tan \& Salama 2005). See Fig. 4 for further details.

occurs near $3266.63 \AA$, yet this is unlikely because of the absence of the stronger band at $3344.16 \AA$.

Part of the laboratory spectrum of benzo[ghi]perylene showing two broad vibrational features at $3501.76 \AA$ and $3512.15 \AA$ (Tan \& Salama 2005) and the corresponding UVES spectra are shown in Fig. 8. The two vibrational bands belong to the $S_{2} \leftarrow$ $S_{0}$ transition. The coincidence of various stellar absorption lines in this wavelength region renders the detection of the relatively broad absorption bands of benzo[ghi]perylene very difficult. The spectra of the hotter stars show He I absorptions near $3498.7 \AA$, $3502.4 \AA$, and $3512.5 \AA$, which coincide with the absorption wavelengths of benzo[ghi]perylene. Other photospheric lines in this region are Ne II (3503.6 $⿱$ A) and Cr vII (3498.9 $\AA$ ). The cooler stars show Ni II (3514.0 $\AA$ ) absorption with shallow Cr II $(3511.8 \AA)$ and He I (3512.5 $\AA$ ). The stellar models do not reproduce the observed spectra near these wavelengths with sufficient accuracy, and some residuals exist near $3514 \AA$ where benzo[ghi]perylene absorptions are expected. The unreddened archival stars of similar spectral type show identical photospheric line profiles with broad wings, which leads us to reject possible contributions from benzo[ghi]perylene to the residuals seen near $3514 \AA$. The strong benzo[ghi]perylene origin band of the $S_{2} \leftarrow S_{0}$ transition occurs near $3685 \AA$ and falls into a spectral region which is dominated by strong photospheric hydrogen absorption lines converging toward the Balmer $\infty \rightarrow 2$ series limit at $3646 \AA$. Because of the strong photospheric Balmer lines, a search of the origin band of the $S_{2} \leftarrow S_{0}$ transition of benzo[ghi]perylene toward early type supergiants is hopeless.

Our UVES spectra are rather noisy indeed at wavelengths below $3210 \AA$ A where the $S_{2} \leftarrow S_{0}$ absorptions of pyrene occur. The signal-to-noise ratio of our spectra is smaller than 100 in general, yet the spectra are still useful as the pyrene transitions

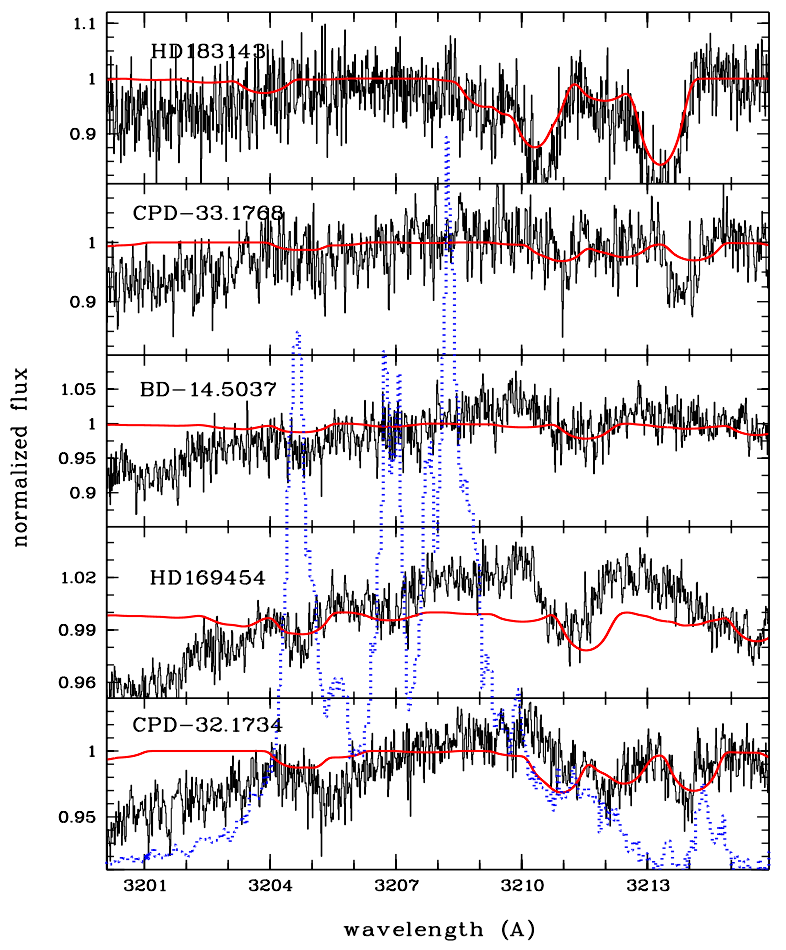

Fig. 9. UVES spectra in the region where the origin of the $S_{2} \leftarrow S_{0}$ transition of pyrene (dotted blue curve) occurs (Rouillé et al. 2004). See Fig. 4 for further details.

exhibit large oscillator strengths. As mentioned in Sect. 2, the interaction of the $S_{2}$ state of pyrene with the vibrational manifold of the $S_{1}$ state gives rise to groups of bands instead of isolated features. Figure 9 shows the group of bands of strong intensities that appear between 3204-3209 $\AA$ in the place of the origin band of $S_{2} \leftarrow S_{0}$ (Rouillé et al. 2004). The UVES data reveal faint stellar absorption lines due to Fe III (3204.8 $⿱$ A) and He I ( $3211.6 \AA)$ in the spectra of the hotter stars. The stellar spectra reveal no signature that correspond to the group of pyrene bands plotted in Fig. 9. Figure 10 compares another group of bands between 3163-3169 $\AA$ which correspond to the excitation of an in-plane CCC bending mode together with the $S_{2}$ electronic state of pyrene. These bands coincide with the stellar

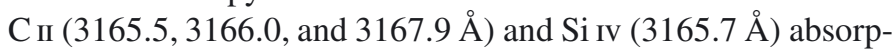
tion lines, which makes a detection of pyrene in this wavelength region difficult, even in spectra of significantly higher $S / N$. Thus, the 3204-3209 A region shown in Fig. 9 with its few and faint stellar lines appears more promising to search for interstellar pyrene. In addition, the pyrene bands in this wavelength region are stronger than between 3163-3169 A.

Figure 11 presents the spectral region where the strongest bands of the $S_{\beta} \leftarrow S_{0}$ transition of hexabenzocoronene is expected. The spectral profile for hexabenzocoronene (dashed blue line) was measured in an Ne matrix and shifted to the expected gas phase position following the method described in Sect. 2.2. We are not able to provide absorption cross sections for hexabenzocoronene because our laboratory data does not reveal the number of individual gas phase bands that form the broad structures seen in Fig. 11. A simulated low-temperature gas phase spectrum (dotted blue curve) that can be compared with the UVES spectra is also shown in Fig. 11. It represents two bands at the gas phase positions predicted for the strongest peaks in the $S_{\beta} \leftarrow S_{0}$ transition of hexabenzocoronene, i.e. at 3344 


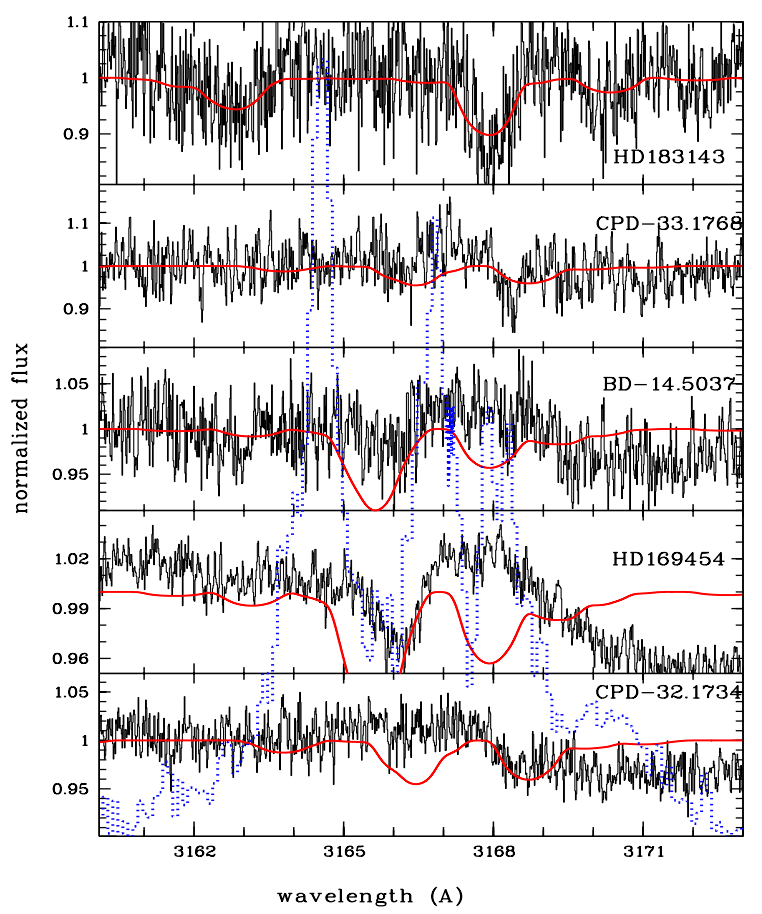

Fig. 10. UVES spectra of another region of the $S_{2} \leftarrow S_{0}$ transition of pyrene (dotted blue curve) involving electronic and vibrational excitation (Rouillé et al. 2004). See Fig. 4 for further details.

and $3369 \AA$. The synthetic bands are represented in terms of Lorentzian profiles, where the ratios of their widths and their heights are obtained from the spectrum of hexabenzocoronene measured in the Ne matrix. We have chosen to fix the widths to 5 and $7 \mathrm{~cm}^{-1}$ because these values are typical of the bandwidths observed in the jet-cooled spectrum of the $S_{2} \leftarrow S_{0}$ transition of benzo[ghi]perylene (Tan \& Salama 2005). This transition is affected by an interaction between electronic states (Tan \& Salama 2005) like the $S_{\beta} \leftarrow S_{0}$ transition of hexabenzocoronene (Rouillé et al. 2009). As stated in Sect. 2.2, groups of bands, not just a pair, are actually expected in the low-temperature gas phase spectrum of hexabenzocoronene in the region covered by Fig. 11. Our UVES spectra do not reveal features resembling the simulated bands of hexabenzocoronene.

We have investigated all archival spectra for potential PAH absorptions. The archival spectra were obtained toward stars with significantly lower reddening, in general, and consequently, the $S / N$ ratio in the blue part of the spectrum is higher than in our spectra. None of the archival spectra reveal traces of the PAHs studied here. This is illustrated in Figs. 12 and 13, which cover the spectral region where the two groups of bands of pyrene studied here occur. These two pyrene bands have relatively large oscillator strengths (cf. Sect. 2 and Table 1), so relatively small column densities of pyrene become detectable. None of the archival spectra show signatures of interstellar pyrene, however. The upper limits in the equivalent widths of pyrene are nevertheless used to constrain the abundance of pyrene in translucent material, as will be demonstrated in the following section.

\subsection{Constraints of PAH column densities in translucent material}

Our UVES spectra together with the laboratory data may be used to infer upper limits in the column densities of

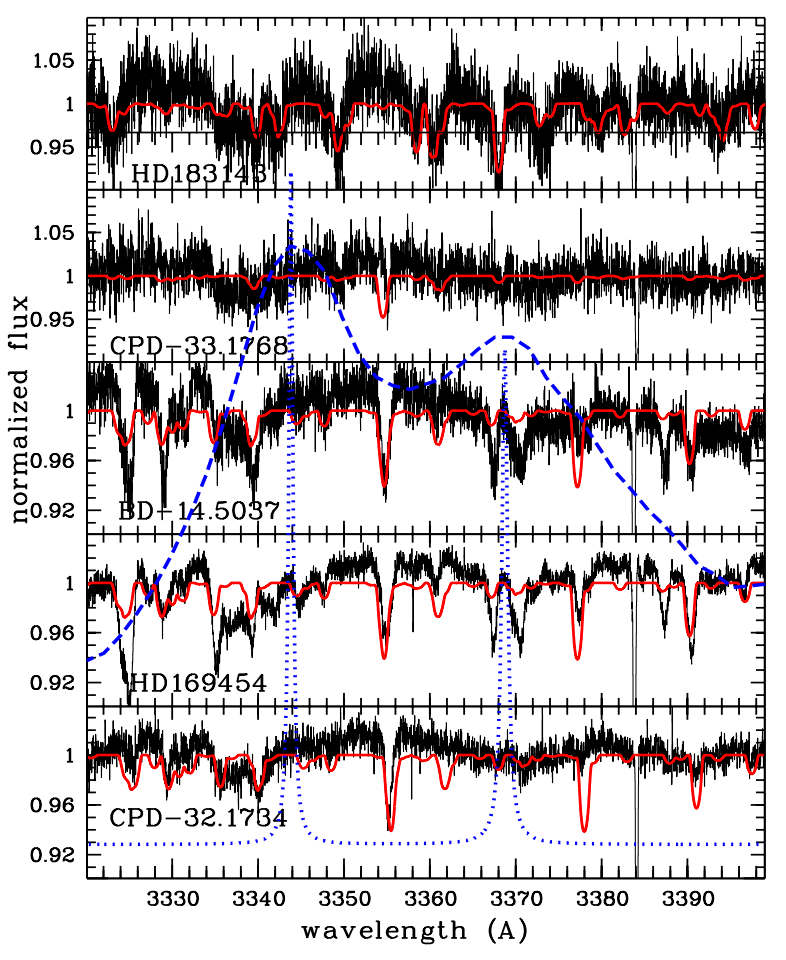

Fig. 11. UVES spectra covering the region where the strongest absorption peaks in the $S_{\beta} \leftarrow S_{0}$ transition of hexabenzocoronene are expected. The dashed blue curve represents the hexabenzocoronene spectrum as measured in an Ne matrix and shifted to the expected gas phase position following the method described in Sect. 2.2. The two narrow bands, plotted as dotted blue curve, are synthetic low-temperature gas phase profiles drawn at their predicted gas phase positions (see text). The sharp absorption feature at $3384 \AA$ is due to interstellar Ti II. See Fig. 4 for further details.

anthracene, phenanthrene, pyrene, 2,3-benzofluorene, and benzo[ghi]perylene. In cases where a sought absorption band is not detected, an upper limit in the equivalent width $W_{\lambda}$ of the absorption band is given by $W_{\lambda}=3 \sqrt{M} \Delta \lambda /(S / N)$, where $\Delta \lambda$ is the spectral width of a resolution element, $M$ is the number of resolution elements over which the sought absorption band spreads, and $S / N$ is the signal-to-noise ratio near the expected absorption feature (see Bohlin et al. 1983, for a comprehensive treatment of error propagation in absorption line measurements). The equivalent width $W_{\lambda}$ of an interstellar absorption line is defined in terms of $W_{\lambda}=\int\left(F_{\mathrm{c}}-F_{\lambda}\right) / F_{\mathrm{c}} \mathrm{d} \lambda$ where $F_{\lambda}$ is the measured flux level at wavelength $\lambda$ and where $F_{\mathrm{c}}$ is the flux in the continuum adjacent to the absorption line. The equivalent width is related to the optical depth $\tau(\lambda)$ according to

$W_{\lambda}=\int\left(1-\mathrm{e}^{-\tau(\lambda)}\right) \mathrm{d} \lambda$

which, for low optical depths $\tau(\lambda) \ll 1$, is expressed as

$W_{\lambda}=\int \tau(\lambda) \mathrm{d} \lambda=N \int \sigma(\lambda) \mathrm{d} \lambda$.

$N$ is the number of absorbers per $\mathrm{cm}^{2}$ and $\sigma(\lambda)$ is the absorption cross section.

Our UVES spectra were obtained with a spectral resolution of $R=\lambda / \Delta \lambda=80000$. For simplicity, we adopt a constant width of $\Delta \lambda=45 \mathrm{~m} \AA$ per resolution element over the entire wavelength range studied here. Table 3 summarizes the central 


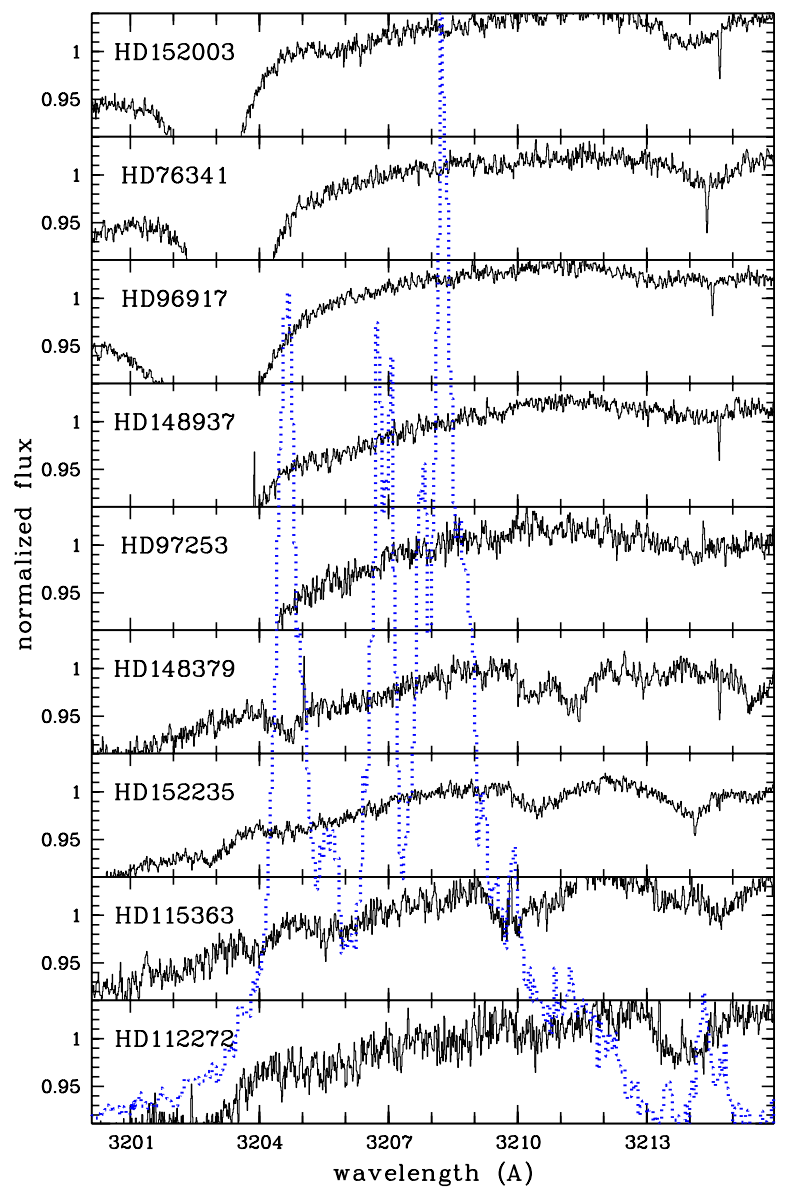

Fig. 12. Archival spectra covering the region where the origin of the $S_{2} \leftarrow S_{0}$ transition of pyrene occurs (Rouillé et al. 2004). See Fig. 4 for further details.

wavelengths and the corresponding values of $3 \sqrt{M} \Delta \lambda$ for each of the sought absorption bands, and the corresponding signalto-noise ratio $S / N$, the upper limits in $W_{\lambda}$, and the upper limits in the column densities $N$ toward HD 169454 , BD $-14^{\circ} 5037$, $\mathrm{CPD}-33^{\circ} 1768, \mathrm{CPD}-32^{\circ} 1734$, and HD 183143.

Our data indicates maximal column densities of anthracene, pyrene, and benzo[ghi]perylene of a few $10^{12} \mathrm{~cm}^{-2}$ toward the heavily reddened programme stars of our UVES sample. Toward the stars retrieved from the archive, upper column densities in pyrene are below values of $10^{12} \mathrm{~cm}^{-2}$. Using the empirical relations between the $\mathrm{CH}$ and $\mathrm{H}$ column densities (Mattila 1986), the upper limits in the PAH column densities and the observed CH column densities (cf. Sect. 4.3) are used to infer upper limits in the fractional abundances of $f(\mathrm{PAH})=N(\mathrm{PAH}) / N(\mathrm{H})=1.1 \times 10^{-8} N(\mathrm{PAH}) / N(\mathrm{CH})$. Our observations indicate values of $f$ (anthracene) $\leq 1.5-2 \times$ $10^{-10}$ toward HD $169454, \mathrm{BD}-14^{\circ} 5037, \mathrm{CPD}-32^{\circ} 1734$, and CPD $-33^{\circ} 1768$, and $f$ (anthracene) $\leq 10^{-9}$ toward HD 183143 . The upper limits in the fractional abundances of pyrene and 2,3-benzofluorene are $\leq 5 \times 10^{-10}$ toward HD 169454 and $\mathrm{BD}-14^{\circ} 5037$ and $\leq 3.5 \times 10^{-9}$ toward the remaining three stars. Fractional abundances of pyrene toward the more diffuse lines of sight traced by the archival stars are below a few times $10^{-10}$. For phenanthrene, which has a relatively small oscillator strength in the transitions studied here (cf. Table 1), upper limits in the fractional abundances are a few $10^{-8}$. Our values may be compared to fractional abundances relative to $\mathrm{H}_{2}$ derived for other complex molecules from radio telescope observations.

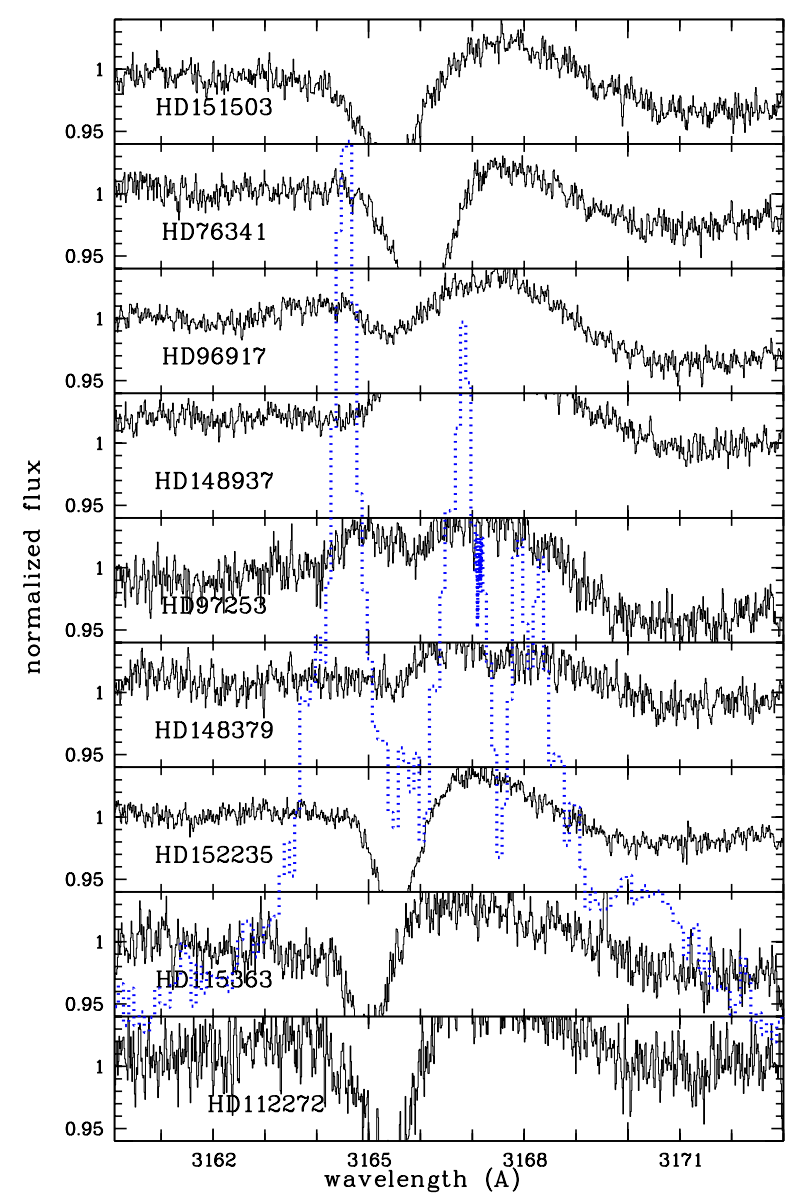

Fig. 13. Archival spectra covering a $S_{2} \leftarrow S_{0}$ transition of pyrene involving electronic and vibrational excitation (Rouillé et al. 2004). See Fig. 4 for further details.

Marcelino et al. (2007) report a relative abundance of $4 \times 10^{-9}$ for propylene detected toward the dark cloud TMC-1 while Belloche et al. (2009) derived abundances for ethyl formate and $n$-propyl cyanide observed toward Sgr B2(N) of 3.6 $\times 10^{-9}$ and $1.0 \times 10^{-9}$, respectively.

\subsection{Interstellar molecular absorption lines}

Our UVES spectra contain various interstellar absorption lines which are presented for consistency checks with previous results.

$\mathrm{OH}^{+}$. Our spectrum obtained toward CPD $-32^{\circ} 1734$ contains an absorption line near $3584 \AA$ which we assign to the interstellar $R_{11}(0)\left(\lambda_{\text {air }}=3583.769 \AA\right)$ line of the $(0,0)$ band of the $A^{3} \Pi_{i}-X^{3} \Sigma^{-}$system of $\mathrm{OH}^{+}$. The relevant part of the spectrum is reproduced in Fig. 14, together with the spectra of HD 169454 and $\mathrm{BD}-14^{\circ} 5037$. Using the band oscillator strength of $f_{00}=$ $3.0764 \times 10^{-3}$ given by de Almeida \& Singh (1981), we convert the measured equivalent width of $W_{\lambda}=4 \mathrm{~m} \AA$ into an $\mathrm{OH}^{+}$column density of $N\left(\mathrm{OH}^{+}\right)=11 \times 10^{12} \mathrm{~cm}^{-2}$. The $\mathrm{OH}^{+} \mathrm{ab}-$ sorption line appears at a heliocentric velocity of $+35.4 \mathrm{~km} \mathrm{~s}^{-1}$ which is consistent with the average velocities of interstel$\operatorname{lar} \mathrm{CN}\left(V_{\text {hel }}=+37.5 \mathrm{~km} \mathrm{~s}^{-1}\right), \mathrm{CH}^{+}\left(V_{\text {hel }}=+35.6 \mathrm{~km} \mathrm{~s}^{-1}\right)$, and $\mathrm{CH}\left(V_{\text {hel }}=+35.6 \mathrm{~km} \mathrm{~s}^{-1}\right)$ toward that line of sight (cf. Table 4).

The $\mathrm{OH}^{+}$radical has only recently been discovered in the interstellar medium. Wyrowski et al. (2010) detected the 
R. Gredel et al.: Abundances of PAHs in the ISM: confronting observations with experimental results

Table 3. Upper limits of large molecules toward the lines of sight studied here.

\begin{tabular}{|c|c|c|c|c|c|c|c|c|c|c|c|c|c|c|c|c|}
\hline \multirow[b]{2}{*}{$\lambda_{\text {air }}$} & \multirow[b]{2}{*}{$3 \sqrt{M} \Delta \lambda$} & \multicolumn{3}{|c|}{ HD 169454} & \multicolumn{3}{|c|}{$\mathrm{BD}-14^{\circ} 5037$} & \multicolumn{3}{|c|}{$\mathrm{CPD}-33^{\circ} 1768$} & \multicolumn{3}{|c|}{$\mathrm{CPD}-32^{\circ} 1734$} & \multicolumn{3}{|c|}{ HD 183143} \\
\hline & & $S / N$ & $W_{\lambda}$ & $N_{\max }{ }^{a}$ & $S / N$ & $W_{\lambda}$ & $N_{\max }^{a}$ & $S / N$ & $W_{\lambda}$ & $N_{\max }{ }^{a}$ & $S / N$ & $W_{\lambda}$ & $N_{\max }{ }^{a}$ & $S / N$ & $W_{\lambda}$ & $N_{\max }^{a}$ \\
\hline anthracene & & & & & & & & & & & & & & & & \\
\hline $\begin{array}{l}3610.74 \\
\text { phenanthrene }\end{array}$ & 371 & 230 & 1.6 & 0.8 & 113 & 3.3 & 1.7 & 81 & 4.6 & 2.4 & 143 & 2.6 & 1.4 & 69 & 5.4 & 2.8 \\
\hline $\begin{array}{l}3409.21 \\
\text { pyrene }\end{array}$ & 284 & 165 & 1.7 & 105 & 97 & 2.9 & 180 & 62 & 4.6 & 285 & 145 & 1.9 & 118 & 53 & 5.3 & 330 \\
\hline 3208.22 & 1493 & 70 & 21 & 2.3 & 37 & 40 & 4.4 & 23 & 65 & 7.2 & 51 & 29 & 3.2 & 23 & 65 & 7.1 \\
\hline 2,3-benzofluo & iorene & & & & & & & & & & & & & & & \\
\hline $\begin{array}{l}3344.16 \\
\text { benzo[ghi]per }\end{array}$ & $\begin{array}{l}270 \\
\text { erylene }\end{array}$ & 117 & 2.3 & 2.7 & 88 & 3.1 & 3.7 & 47 & 5.7 & 6.8 & 117 & 2.3 & 2.8 & 41 & 6.6 & 7.9 \\
\hline 3512.15 & 1822 & 206 & 8.8 & 8.8 & 125 & 14.6 & 14.6 & 82 & 22 & 22 & 165 & 11 & 11 & 76 & 24 & 24 \\
\hline 3501.76 & 1465 & 206 & 7.1 & 12.8 & 125 & 11.7 & 21 & 82 & 18 & 32 & 165 & 8.9 & 16 & 76 & 19 & 34 \\
\hline
\end{tabular}

Notes. ${ }^{(a)}$ Upper limits $N_{\max }$ in units of $10^{12} \mathrm{~cm}^{-2}$.

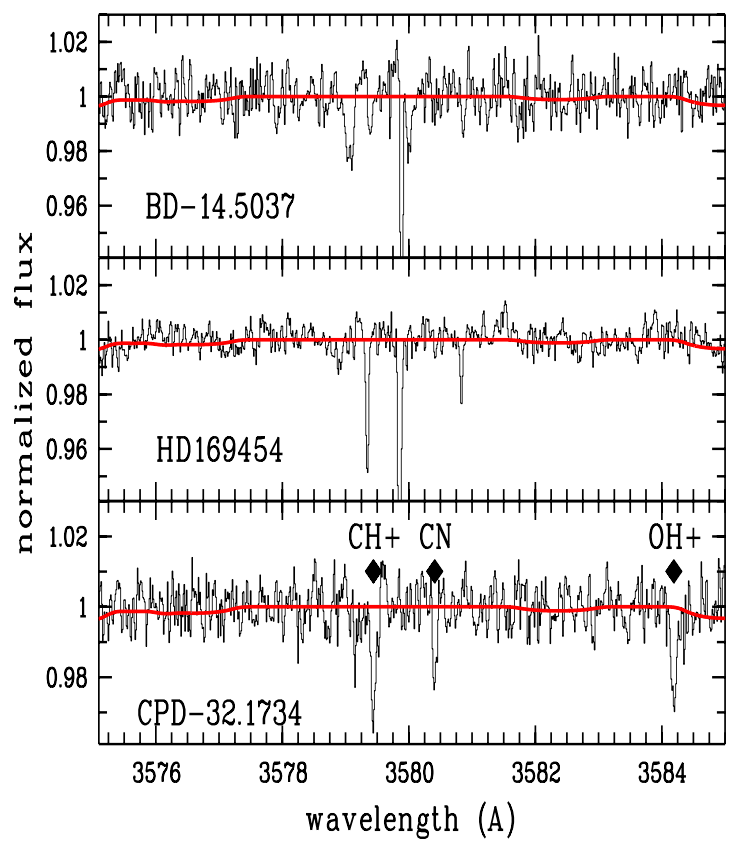

Fig. 14. Detection of the interstellar $\mathrm{OH}^{+}$radical in the spectrum toward CPD $-32^{\circ} 1734$. Spectra toward HD 169454 and BD $-14^{\circ} 5037$ are shown as well. All spectra are normalized to a continuum level of 1.0 and rebinned to a heliocentric wavelength scale. The $\mathrm{OH}^{+}, \mathrm{CH}^{+}$, and $\mathrm{CN}$ absorption lines toward $\mathrm{CPD}-32^{\circ} 1734$ are explicitly identified. Synthetic stellar spectra obtained from the models of Gummersbach \& Kauffer (1993) are shown as red continuous curves.

$\mathrm{OH}^{+} N=1-0, J=0-1$ absorption line toward the strong continuum source Sgr B2 using APEX at sub-mm wavelengths, and Gerin et al. (2010) report the detection of $\mathrm{OH}^{+}$absorption toward G10.6-04 (W31C) using HIFI on HERSCHEL. Optical absorption lines arising from interstellar $\mathrm{OH}^{+}$have been searched by de Almeida \& Singh (1981) without success. Optical detections of $\mathrm{OH}^{+}$are reported by Krełowski et al. (2010a). Using the relations of Mattila (1986), the observed columns of $\mathrm{OH}^{+}$and $\mathrm{CH}$ are used to infer a fractional abundance of $N\left(\mathrm{OH}^{+}\right) / N(\mathrm{H})=$ $1.1 \times 10^{-8} N\left(\mathrm{OH}^{+}\right) / N(\mathrm{CH})=9 \times 10^{-10}$, where $N(\mathrm{H})=2 N\left(\mathrm{H}_{2}\right)$. The fractional abundance of $\mathrm{OH}^{+}$of about $10^{-9} \mathrm{~cm}^{-2}$ is almost two orders of magnitude lower than the abundances inferred toward the massive star forming region W31C (Gerin et al. 2010). The absence of velocity shifts between $\mathrm{OH}^{+}$and the neutral interstellar molecules (cf. Table 4) argues against a production of
$\mathrm{OH}^{+}$in shocks, as proposed by de Almeida (1990). Interstellar shocks have been ruled out as the general production site of $\mathrm{CH}^{+}$ (Gredel et al. 1993).

$\mathrm{NH}$. Interstellar NH has been discovered by Meyer \& Roth (1991). From their measurements of the $R_{1}(0) \quad\left(\lambda_{\text {air }}=\right.$ $3358.0525 \AA)$ and ${ }^{R} Q_{21}(0)\left(\lambda_{\text {air }}=3353.9235 \AA\right)$ lines of the $(0,0)$ band of the $A^{3} \Pi-X^{3} \Sigma$ system, the authors inferred column densities of $1 \times 10^{12} \mathrm{~cm}^{-2}$ and $2.7 \times 10^{12} \mathrm{~cm}^{-2}$ toward $\zeta$ Per and HD 27778, respectively. Both lines are detected in our spectrum of HD 169454 and are marginally present toward CPD $-32^{\circ} 1734$. We use oscillator strengths of $f=0.0041$ for the $R_{1}(0)$ and $f=0.0024$ for the ${ }^{R} Q_{21}(0)$ line (Meyer \& Roth $1991)$ to infer a column density of $N(\mathrm{NH})=7.6 \times 10^{12} \mathrm{~cm}^{-2}$ toward HD 169454. The column density is in good agreement with the value of $6.7 \times 10^{12} \mathrm{~cm}^{-2}$ given recently by Weselak et al. (2009a) in their comprehensive analysis of interstellar NH. An NH column density of $N(\mathrm{NH}) \approx 2.4 \times 10^{12} \mathrm{~cm}^{-2}$ is inferred toward $\mathrm{CPD}-32^{\circ} 1734$. Toward both lines of sight, the radial velocities of the $\mathrm{NH}$ absorption lines agree with those of $\mathrm{CH}, \mathrm{CH}^{+}$, and $\mathrm{CN}$.

CN. The $R(1)\left(\lambda_{\text {air }}=3579.453 \AA\right), R(0)\left(\lambda_{\text {air }}=3579.963 \AA\right)$, and $P(1)\left(\lambda_{\text {air }}=3580.937 \AA\right)$ lines of the $(1,0)$ band of the $B^{2} \Sigma-X^{2} \Sigma$ system of $C N$ are detected toward HD 169454, and the $\mathrm{R}(0)$ line is detected toward $\mathrm{BD}-14^{\circ} 5037, \mathrm{CPD}-32^{\circ} 1734$, and CPD $33^{\circ} 1768$. We use the molecular parameters given by Meyer et al. (1989) to infer interstellar CN abundances of $N(J=$ $0)=2.4 \times 10^{13} \mathrm{~cm}^{-2}$ and $N(J=1)=1.2 \times 10^{13} \mathrm{~cm}^{-2}$ toward HD 169454, in the limit of Doppler- $b$ values of $b \rightarrow \infty$. The ratio in the population densities corresponds to an excitation temperature of $T_{\mathrm{ex}}=2.9 \mathrm{~K}$. The value of $N(J=0)$ inferred here is consistent with the value of $N(J=0)=3 \times 10^{13} \mathrm{~cm}^{-2}$ obtained for a Dopper- $b$ value of $b_{\mathrm{vr}}=0.45 \mathrm{~km} \mathrm{~s}^{-1}$ (Gredel et al. $1991)$, where $b_{\mathrm{vr}}$ was obtained from an analysis of CN absorption lines arising in the violet and red systems. The $\mathrm{CN}$ column densities in $J=0$ toward $\mathrm{BD}-14^{\circ} 5037$, CPD $-32^{\circ} 1734$, and CPD $-33^{\circ} 1768$ (cf. Table 4) are consistent with the measurements of Gredel et al. (1991) and Gredel et al. (2002) toward those lines of sight.

$\mathrm{CH}$. We report the detection of the $R_{2}(1)\left(\lambda_{\text {air }}=3137.576 \AA\right)$ and ${ }^{P} Q_{12}(1)\left(\lambda_{\text {air }}=3145.996 \AA\right)$ lines and of the $\left[Q_{2}(1)+\right.$ $Q_{\left.R_{12}(1)\right]}\left(\lambda_{\text {air }}=3143.183 \AA\right)$ unresolved line blend of the 
$(0,0)$ band of the $C^{2} \Sigma^{+}-X^{2} \Pi$ system of $\mathrm{CH}$. In addition, the $\left[{ }^{Q} R_{12}(1)+Q_{12}(1)\right]\left(\lambda_{\text {air }}=3633.29 \AA\right)$ line blend and the ${ }^{P} Q_{12}(1)\left(\lambda_{\text {air }}=3636.27 \AA\right)$ line of the $(1,0)$ band of the $B^{2} \Sigma^{-}-X^{2} \Pi$ system are detected. The measured equivalent widths of the lines arising in the $\mathrm{C}$-X system are converted into column densities using the molecular parameters and the method of Lien (1984). For the oscillator strength of the $(1,0)$ band of the B-X system, we scale the value of the $(0,0)$ band of Lien (1984) using the ratio of 7.8 of the band oscillator strengths of the $(0,0)$ and $(1,0)$ bands as inferred from the lifetime measurements of Brooks \& Smith (1974). The observed CH absorption lines and line blends arise from the lower or upper component of the $J=1 / 2 \Lambda$-doublet. In Table 4 , we list total $\mathrm{CH}$ column densities $N_{\text {tot }}$, which are obtained under the assumption that $N_{\text {tot }}=2 N_{\mathrm{l}} ; 2 N_{\mathrm{u}}$, where $N_{\mathrm{l}}$ and $N_{\mathrm{u}}$ are the column densities in the lower and upper $\Lambda$ components, respectively. For the purpose of the present consistency check, we assume that all lines are optically thin, and ignore a detailed curve of growth analysis.

$\mathrm{CH}^{+}$. The $R(0)$ line $\left(\lambda_{\text {air }}=3745.307 \AA\right)$ of the $(2,0)$ band of the $A^{1} \Pi-X^{1} \Sigma^{+}$system of $\mathrm{CH}^{+}$is detected toward all five lines of sight studied here, and the $R(0)$ line of the $(3,0)$ band $\left(\lambda_{\text {air }}=\right.$ $3579.021 \AA)$ is detected toward HD 169454, BD $-14^{\circ} 5037$, $\mathrm{CPD}-32^{\circ} 1734$, and $\mathrm{CPD}-33^{\circ} 1768$. We use the molecular parameters given by Weselak et al. (2009b) to infer $\mathrm{CH}^{+}$column densities ranging from $N\left(\mathrm{CH}^{+}\right)=1.6 \times 10^{13} \mathrm{~cm}^{-2}$ toward HD 169454 to $N\left(\mathrm{CH}^{+}\right) \approx 4 \times 10^{13} \mathrm{~cm}^{-2}$ toward BD $-14^{\circ} 5037$, CPD $-32^{\circ} 1734$, and CPD $-33^{\circ} 1768$.

\section{Discussion}

\subsection{The long and eventful history of DIB candidates}

Over the last decades, a large variety of potential DIB carriers has been proposed, either because laboratory spectra revealed coincidences with absorption features in astronomical spectra or because theoretical calculations predicted molecular transition wavelengths that matched those of observed DIBs. For instance, Tulej et al. (1998) proposed $\mathrm{C}_{7}^{-}$based on a few coincidences between bound-bound electronic transitions of $\mathrm{C}_{7}^{-}$and five DIB absorption wavelengths. The proposition is no longer generally accepted (McCall et al. 2001), as the exact wavelengths and absorption profiles of the $\mathrm{C}_{7}^{-}$lines do not match the interstellar profiles closely enough. A number of close agreements in the absorption wavelengths of neutral carbon chain molecules and their cations with DIB absorptions were found by Motylewski et al. (2000), yet in all cases small but significant shifts between the laboratory and the interstellar absorption features remained. These shifts could not be explained by radial velocity shifts of the interstellar material nor could they be traced to calibration issues in the astronomical data. $\mathrm{The}^{\mathrm{H}} \mathrm{HC}_{4} \mathrm{H}^{+}$diacetylene cation has recently been purported as a DIB carrier (Krełowski et al. 2010b). The authors averaged a large number of stellar spectra of different stars in order to improve the $S / N$-ratio. The average spectrum reveals a faint absorption feature near $5069 \AA$ which Krełowski et al. (2010b) assign to a previously undetected diffuse interstellar band. The gas-phase spectra obtained by Motylewski et al. (2000) shows a prominent absorption band of $\mathrm{HC}_{4} \mathrm{H}^{+}$at $5069 \AA$, which matches exactly the position of the $5069 \AA$ feature. However, the $\mathrm{HC}_{4} \mathrm{H}^{+}$band profile obtained from the laboratory data deviates significantly from the $5069 \AA$ feature, and it is difficult to see how a convolved $\mathrm{HC}_{4} \mathrm{H}^{+}$band profile, assuming typical Doppler- $b$ broadening of

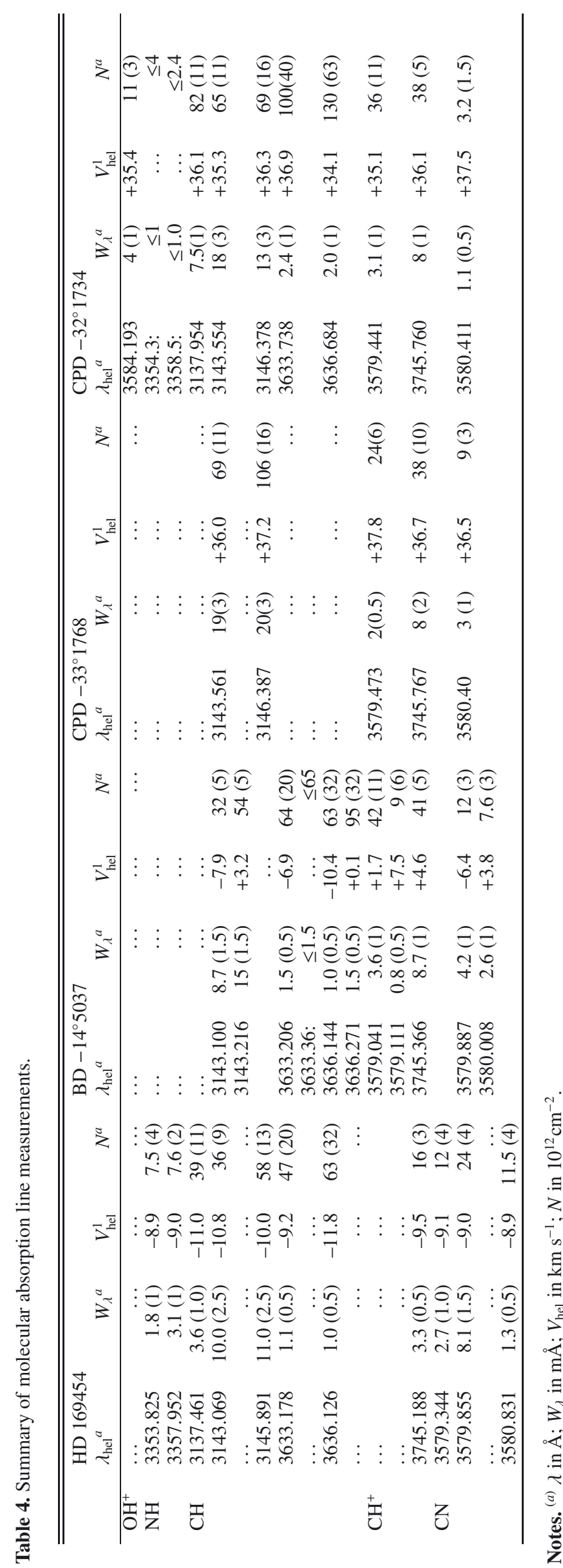


the rotational lines and macroscopic turbulent gas motions in translucent clouds, can be brought into agreement with the profile of the $5069 \AA$ A absorption feature reported by Krełowski et al. (2010b).

In a similar fashion, the recent evidence provided by Maier et al. (2011) that $l-\mathrm{C}_{3} \mathrm{H}_{2}$ is responsible for the $5450 \AA$ and $4881 \AA$ DIBs requires some scrutiny. While the authors convincingly assign two absorption features near $4887 \AA$ and $5450 \AA$ seen in their laboratory CRDS spectra to the $2_{0}^{1}$ and $2_{0}^{2}$ transitions in the $B^{1} \mathrm{~B}_{1} \leftarrow X^{1} \mathrm{~A}_{1}$ system of $l-\mathrm{C}_{3} \mathrm{H}_{2}$, the agreement with interstellar DIBs is good for the $5450 \AA$ line only. The $4887 \AA$ transition of $l-\mathrm{C}_{3} \mathrm{H}_{2}$ does not match the exact shape and position of the $4881 \AA$ DIB, unless it is assumed that several DIB carriers contribute to this feature. More critically, Ne-matrix spectra also reveal an $l-\mathrm{C}_{3} \mathrm{H}_{2}$ absorption feature which arises from the $20_{0}^{1} 4_{0}^{1}$ transition, which is about a factor of $2-3$ fainter than the $4887 \AA$ and $5450 \AA$ transitions of $l-\mathrm{C}_{3} \mathrm{H}_{2}$. The strength of the $2{ }_{0}^{1} 4{ }_{0}^{1}$ transition is below the detection limit of the CRDS experiments presented by Maier et al. (2011) and an accurate gas phase wavelength is not available, yet its position is predicted to be near $5173 \AA$. Maier et al. (2011) did search their spectra for an interstellar absorption band near $5173 \AA$, yet they note that the presence of various stellar absorption lines hampered their effort. The high $S / N$ spectrum presented by Hobbs et al. (2009) toward HD 183143 does show broad and prominent DIB absorptions near $4881 \AA$ and $5450 \AA$. The latter spectrum is relatively clean in the 5165-5180 $\AA$ region, yet it does not show the expected signature from the $2{ }_{0}^{1} 4_{0}^{1}$ transition of $l-\mathrm{C}_{3} \mathrm{H}_{2}$ in this wavelength interval.

Other molecular cations and anions have served as prominent candidates of DIB carriers in the past. Matrix isolation experiments of $\mathrm{C}_{60}^{+}$revealed absorption features near the $9577 \AA$ and $9632 \AA$ DIBs (Foing \& Ehrenfreund 1997), however a confirmation of the $\mathrm{C}_{60}^{+}$gas phase wavelengths based on CRDS spectra is not yet available. That work supported the general proposition that PAH cations are responsible for DIB absorptions (Salama et al. 1996, 1999). Jet-cooled absorption spectra measured in the laboratory (Romanini et al. 1999; Biennier et al. 2003, 2004; Sukhorukov et al. 2004; Tan \& Salama 2006) were subsequently obtained, and interstellar spectra obtained toward the Perseus molecular cloud complex did reveal weak features that coincide with absorption bands of the naphthalene and anthracene cations (Iglesias-Groth et al. 2008, 2010). In the case of the anthracene cation, the CRDS spectrum of Sukhorukov et al. (2004) reveals an absorption at $7085.7 \AA$ which has an $F W H M$ of $47 \AA$. While the spectrum of Iglesias-Groth et al. (2010) may show an interstellar absorption band near that wavelength, the width of the purported interstellar absorption feature is significantly smaller than $47 \AA$.

Potential DIB carriers have also been proposed from theory. With rotational contour fitting, Kerr et al. (1996) were able to reproduce the structure seen in the $6614 \AA$ and $5797 \AA$ DIBs assuming that they are due to planar oblate symmetric top molecules, such as large carbon ring molecules with 14-30 atoms, rather than PAHs. An excellent agreement was found between the calculated absorption wavelength of the $0_{0}^{0}$ band of the ${ }^{1} B_{1}-X^{1} A^{\prime}$ electronic system of $\mathrm{CH}_{2} \mathrm{CN}^{-}$and the $8037 \AA$ DIB (Cordiner \& Sarre 2007). However, the calculated absorption wavelength needs to be confirmed by gas phase laboratory experiments. It is also noted that the match between the calculated $\mathrm{CH}_{2} \mathrm{CN}^{-}$absorption and the $8037 \AA$ DIB is good for an assumed rotational excitation temperature of $2.7 \mathrm{~K}$ and a Doppler- $b$ value of $16-33 \mathrm{~km} \mathrm{~s}^{-1}$. At smaller $b$-values, the rotational structure of $\mathrm{CH}_{2} \mathrm{CN}^{-}$should be resolved in the astronomical spectra, in disagreement with the observed broad and diffuse absorption near $8037 \AA$. It is also noted that at a nominal ortho to para ratio of $3: 1$, the calculated spectrum of $\mathrm{CH}_{2} \mathrm{CN}^{-}$does not match the observed spectra. Similarly, a recent claim that proflavine and its anion, cation, and di-cation are responsible for the $4066 \AA, 4363 \AA, 4175 \AA$, and $5259 \AA$ DIBs (Bonaca \& Bilalbegović 2010) requires verification. The proposition is based on computed optical absorption wavelengths using pseudo-potential density functional theory methods. The matches in wavelengths are marginal at most, and need to be confirmed by laboratory experiments. In any case, as pointed out at the end of Sect. 2.1, extreme care must be taken if computational results on electronic transitions are used for the interpretation of astronomical observations, due to the large uncertainties still involved in present-day calculations.

\subsection{Fractional $P A H$ abundances in translucent molecular clouds}

PAHs are believed to be formed in the outflows of carbon-rich AGB stars from where they are deposited into the interstellar medium. The fractional PAH abundance is estimated to be of the order of $f(\mathrm{PAH})=N(\mathrm{PAH}) / N(\mathrm{H})=10^{-7}$ (Habart et al. 2004), assuming some 100 carbon atoms per $\mathrm{PAH}$ molecule. In the diffuse interstellar material, at densities of $n_{\mathrm{H}}=100 \mathrm{~cm}^{-3}$, a temperature of $T=100 \mathrm{~K}$, and fractional electron abundances of $n_{\mathrm{e}}=10^{-4}$, small PAHs such as anthracene, phenanthrene, and pyrene, are rapidly destroyed by the ambient UV radiation (Le Page et al. 2003). The photoionization of PAHs in diffuse clouds is a major heating source (Lepp \& Dalgarno 1988a) and supersedes the grain heating for PAH abundances greater than $2 \times 10^{-7}$. As the visual extinction increases, the abundance of PAHs increases rapidly, as shown by Lepp et al. (1988). The authors postulated a fractional PAH abundance of $10^{-7}$ in an attempt to resolve density discrepancies which persisted for translucent molecular cloud models. The transition between neutral PAHs and their anions and cations is governed by the balance between photoionization and recombination as well as electron attachment and photo-detachment. At visual extinctions of $A_{\mathrm{V}}=1$ mag (which corresponds to a reddening of $E_{B-V}=0.3 \mathrm{mag}$ ), the fractional abundances of neutral PAHs and their anions are comparable and exceed the fractional abundance of the positively charged PAHs by about two orders of magnitude. As the visual extinction increases further, PAHs form a drain to the free electrons for PAH abundances greater than $10^{-8}$, resulting in a substantial reduction in the density of electrons (Lepp \& Dalgarno 1988b). As a result, the molecular ions undergo nondestructive neutralization reactions with the negatively charged PAHs, which leads to a significant increase of the fractional abundances of the carbonbearing molecules.

Based on the infrared emission bands, Allamandola et al. (1985) evaluated the fractional abundance of interstellar PAHs to be $2 \times 10^{-7}$. Later, Allamandola et al. (1989) distinguished between small and large PAHs, which were defined as containing 25 and 300-400 C atoms, respectively. A minimal fractional abundance of $3 \times 10^{-8}$ was obtained for the small PAHs while a value of $8 \times 10^{-9}$ was derived for the large PAHs (Allamandola et al. 1989), corresponding to fractions of a $1-5 \%$ and $0.5 \%$ of elemental carbon locked in small and large PAHs, respectively. Considering a single class of PAHs with a typical size of $50 \mathrm{C}$ atoms, an abundance of about $3 \times 10^{-7}$ was derived by 
Tielens (2008). Of interest to our study is the minimal interstellar fractional abundance of the small PAHs. With the exception of hexabenzocoronene, the molecules we have studied can be considered to belong to the class of small PAHs. The upper limits we have derived for the fractional abundances of anthracene, pyrene, and 2,3-benzofluorene are about two to three orders of magnitude lower than the estimates presented by Tielens (2008). The fractional abundance of phenanthrene is not well constrained from our observations, mainly because the phenanthrene band studied here has a small oscillator strength. We would thus conclude that small and neutral PAHs must occur in a large variety (several 100 species) in translucent clouds, if it is assumed a fraction of several percent of the elemental carbon is locked up in small PAHs. Alternatively, small and neutral PAHs may not be very abundant in translucent material, either because small PAHs carry positive or negative charge or because significantly less elemental carbon is locked up in these molecules.

In their investigation of the ultraviolet interstellar extinction curve using the HST/STIS spectrograph, Clayton et al. (2003) did not detect absorption bands in the 1150-3180 A wavelength region that could be associated with PAHs. The authors concluded that either PAHs are destroyed by the interstellar radiation field, or that they occur in such a large variety that individual absorptions are too weak to be detected.

Concerning the larger PAHs, an upper limit in the column density of hexabenzocoronene of $4 \times 10^{12} \mathrm{~cm}^{-2}$ in translucent molecular material has recently been estimated by Kokkin et al. (2008) from spectra covering the vibronic hexabenzocoronene absorptions in the $\alpha$-band near $4262 \AA$. The $\alpha$-band is, however, not well suited for the search of interstellar hexabenzocoronene, as it is significantly weaker than the $\beta$-band near $3344 \AA$ (Rouillé et al. 2009). Unfortunately, we are not able to constrain the molecular abundance of hexabenzocoronene from our observations, as we were unable to derive absorption cross sections of the $\beta$-band (cf. Sects. 2.1 and 4). We nevertheless propose that searches for interstellar hexabenzocoronene be conducted near $3344 \AA$.

\subsection{Molecular anions in translucent material}

Carbon-chain molecular anions have recently been established as a major constituent of dense material. The detection of $\mathrm{C}_{6} \mathrm{H}^{-}$ in the TMC-1 molecular cloud by McCarthy et al. (2006) marks the discovery of molecular anions in the interstellar medium. That work was followed by the observation of several other anions in interstellar and circumstellar material, e.g. $\mathrm{C}_{4} \mathrm{H}^{-}$ (Cernicharo et al. 2007; Agúndez et al. 2008), $\mathrm{C}_{8} \mathrm{H}^{-}$(Remijan et al. 2007), $\mathrm{C}_{3} \mathrm{~N}^{-}$(Thaddeus et al. 2008), and $\mathrm{CN}^{-}$(Agúndez et al. 2010). The effects of molecular anions on the chemistry of dark clouds have been rediscussed by Millar et al. (2007) and Walsh et al. (2009). The models set up for dark clouds produce large amounts of molecular anions at molecular cloud ages below $10^{5} \mathrm{yr}$. The anion abundances are proportional to the electron abundance and are characterized by a sharp drop by several orders of magnitude as the clouds reach ages of $10^{6} \mathrm{yr}$ or above. The anion to neutral ratio is typically of the order of a few percent.

\subsection{Individual lines of sight}

HD 169454 and BD $-14^{\circ} 5037$. Various studies of the lines of sight toward HD 169454 and BD $-14^{\circ} 5037$ are available in the literature. The $C_{2}$ observations of Gredel \& Muench (1986) revealed the presence of very cold gas toward HD 169454, with gaskinetic temperatures of $T_{\text {kin }}=15 \mathrm{~K}$ and gas densities of $n=$ $400 \mathrm{~cm}^{-3}$. The $\mathrm{C}_{2}$ absorption occurs at heliocentric velocities of $V_{\text {hel }}=-8.5 \mathrm{~km} \mathrm{~s}^{-1}$. Toward $\mathrm{BD}-14^{\circ} 5037$, the $\mathrm{C}_{2}$ absorption occurs in two velocity components at $V_{\text {hel }}=-6.0 \mathrm{~km} \mathrm{~s}^{-1}$ and $V_{\text {hel }}=$ $+4.0 \mathrm{~km} \mathrm{~s}^{-1}$, where the gas in the two velocity components is characterized by temperatures and densities of $T_{\text {kin }}=30 \mathrm{~K}$ and $n=400 \mathrm{~cm}^{-3}$ and $T_{\text {kin }}=50 \mathrm{~K}$ and $n=250 \mathrm{~cm}^{-3}$, respectively. The $\mathrm{CH}$ and $\mathrm{CN}$ absorption lines are resolved into two velocity components at similar heliocentric velocities (cf. Table 4). The $\mathrm{CH}^{+}$absorption profiles are unresolved and appear near heliocentric velocities which are consistent with those of $\mathrm{C}_{2}, \mathrm{CH}$, and CN. Very small Doppler- $b$ values of $b=0.4 \mathrm{~km} \mathrm{~s}^{-1}$ toward both lines of sight were inferred by Gredel et al. (1991) from a comparison of $\mathrm{CN}$ column densities derived from the $(0,0)$ bands of the $\mathrm{CN}$ red and violet systems. A curve of growth analysis using $b=0.4 \mathrm{~km} \mathrm{~s}^{-1}$ resulted in total $\mathrm{CN}$ column densities of $N(\mathrm{CN})=4.2 \times 10^{13} \mathrm{~cm}^{-2}$ and $N(\mathrm{CN})=1.6 \times 10^{13} \mathrm{~cm}^{-2}$ toward HD 169454 and $\mathrm{BD}-14^{\circ} 5037$, respectively, and in very good agreement with the present results. The present data are obtained from absorption lines in the $(1,0)$ band, which are about a factor of 10 weaker than the absorption lines in the $(0,0)$ band. The excellent agreement between both sets of measurements confirms the very small Doppler- $b$ values toward both lines of sight. Using the small Doppler- $b$ values, Gredel et al. (1993) obtained $\mathrm{CH}$ and $\mathrm{CH}^{+}$column densities of $N(\mathrm{CH})=4.4 \times$ $10^{13} \mathrm{~cm}^{-2}$ and $N\left(\mathrm{CH}^{+}\right)=1.8 \times 10^{13} \mathrm{~cm}^{-2}$ toward HD 169454 , and $N(\mathrm{CH})=7.3 \times 10^{13} \mathrm{~cm}^{-2}$ and $N\left(\mathrm{CH}^{+}\right)=6.4 \times 10^{13} \mathrm{~cm}^{-2}$ toward $\mathrm{BD}-14^{\circ} 5037$, again in excellent agreement with the present results.

CPD $-32^{\circ} 1734$ and CPD $-33^{\circ} 1768$. Very large column densities of $\mathrm{C}_{2}, \mathrm{CH}, \mathrm{CH}^{+}$, and $\mathrm{CN}$ have previously been measured toward the heavily reddened supergiants CPD $-32^{\circ} 1734$ and CPD $-33^{\circ} 1768$ in the NGC 2439 association (Gredel et al. 2002, and references therein). From observations of $\mathrm{C}_{2}$, gaskinetic temperatures of $T_{\text {kin }}=85 \mathrm{~K}$, densities of $n>1000 \mathrm{~cm}^{-3}$, and column densities of $N\left(\mathrm{C}_{2}\right) \approx 10^{14} \mathrm{~cm}^{-2}$ were inferred toward both lines of sight. The $\mathrm{CH}$ and $\mathrm{CH}^{+}$column densities reach values of $N\left(\mathrm{CH}^{+}\right)=4.4 \times 10^{13} \mathrm{~cm}^{-2}$ and $N(\mathrm{CH})=9 \times 10^{13} \mathrm{~cm}^{-2}$ toward CPD $-32^{\circ} 1734$ and $N\left(\mathrm{CH}^{+}\right)=6.0 \times 10^{13} \mathrm{~cm}^{-2}$ and $N(\mathrm{CH})=12 \times 10^{13} \mathrm{~cm}^{-2}$ toward CPD $-33^{\circ} 1768$. The column densities and heliocentric velocities obtained here are in excellent agreement with the previously published results.

HD 183143. A comprehensive optical study of the diffuse interstellar bands toward HD 183143 has recently been introduced by Hobbs et al. (2009). The authors present a catalog of 414 DIBs from spectra obtained in the 3900-8100 $\AA$ wavelength region with a signal-to-noise ratio of $S / N \approx 1000$ and a spectral resolution of $R=38000$. Their study forms part of a new survey of DIBs toward $30 \mathrm{OB}$ stars carried out at the Apache Point Observatory at a very high signal-to-noise ratio. Molecular carbon was not detected toward HD 183143 (Gredel 1999), which indicates that the line of sight is characterized by low-density material.

\section{Summary}

We have presented high signal-to-noise $(S / N>100)$ absorption line spectra toward heavily reddened early type supergiants in the 3050-3850 ̊ wavelength region. The spectra are compared 
with laboratory gas-phase spectra of anthracene, phenanthrene, pyrene, 2,3-benzofluorene, and benzo[ghi]perylene. We have developed methods to infer absolute absorption cross sections from the laboratory spectra, which we use to derive upper limits in the column densities and the abundances of these neutral PAHs. Our methods lead to reasonable values for the absorption cross sections, if compared with cross sections derived from ab initio methods. The fractional abundances for the PAHs are below a few times $10^{-10}$ for anthracene, pyrene, and 2,3-benzofluorene. Fractional abundances of benzo[ghi]perylene below a few times $10^{-9}$ and of phenanthrene below a few times $10^{-8}$ are derived. Thus, a large variety of small and neutral PAHs such as anthracene and pyrene is required to explain a canonical value of $10^{-7}$ for the total fractional PAH abundance in the interstellar medium. Alternatively, it may be conlcuded that small and neutral PAHs are suppressed in translucent molecular clouds, either because the small PAHs carry predominantly positive or negative charge or because they do not survive the prevailing physical conditions in such clouds.

Acknowledgements. Discussions with J. Krełowski and with S. Federman are kindly acknowledged. F.H., G.R., and Y.C. acknowledge the financial support of the Deutsche Forschungsgemeinschaft (DFG).

\section{References}

Agúndez, M., Cernicharo, J., Guélin, M., et al. 2008, A\&A, 478, L19 Agúndez, M., Cernicharo, J., Guélin, M., et al. 2010, A\&A, 517, L2 Allamandola, L. J., Tielens, A. G. G. M., \& Barker, J. R. 1985, ApJ, 290, L25 Allamandola, L. J., Tielens, A. G. G. M., \& Barker, J. R. 1989, ApJS, 71, 733 Bagnulo, S., Jehin, E., Ledoux, C., et al. 2003, The Messenger, 114, 10 Banisaukas, J., Szczepanski, J., Vala, M., \& Hirata, S. 2004, J. Phys. Chem. A, 108,3713

Bauschlicher, C. W., Boersma, C., Ricca, A., et al. 2010, ApJS, 189, 341 Belloche, A., Garrod, R. T., Müller, H. S. P., et al. 2009, A\&A, 499, 215 Biennier, L., Salama, F., Allamandola, L. J., \& Scherer, J. J. 2003, J. Chem. Phys., 118, 7863

Biennier, L., Salama, F., Gupta, M., \& O'Keefe, A. 2004, Chem. Phys. Lett., 387, 287

Biktchantaev, I., Samartsev, V., \& Sepiol, J. 2002, J. Lum., 98, 265 Bohlin, R. C., Jenkins, E. B., Spitzer, Jr., L., et al. 1983, ApJS, 51, 277 Bonaca, A., \& Bilalbegović, G. 2010, Chem. Phys. Lett., 493, 33 Bondybey, V. E., Smith, A. M., \& Agreiter, J. 1996, Chem. Rev., 96, 2113 Brooks, N. H., \& Smith, W. H. 1974, ApJ, 194, 513

Cami, J., Sonnentrucker, P., Ehrenfreund, P., \& Foing, B. H. 1997, A\&A, 326, 822

Cami, J., Bernard-Salas, J., Peeters, E., \& Malek, S. E. 2010, Science, 329, 1180 Cernicharo, J., Guélin, M., Agúndez, M., et al. 2007, A\&A, 467, L37 Chako, N. Q. 1934, J. Chem. Phys., 2, 644

Clayton, G. C., Gordon, K. D., Salama, F., et al. 2003, ApJ, 592, 947

Cordiner, M. A., \& Sarre, P. J. 2007, A\&A, 472, 537

de Almeida, A. A. 1990, Rev. Mex. Astron. Astrofis., 21, 499

de Almeida, A. A., \& Singh, P. D. 1981, A\&A, 95, 383

Duley, W. W., \& Kuzmin, S. 2010, ApJ, 712, L165

Ehrenfreund, P., D’Hendecourt, L., Verstraete, L., et al. 1992, A\&A, 259, 257

Federman, S. R., \& Lambert, D. L. 1992, AJ, 104, 691

Foing, B. H., \& Ehrenfreund, P. 1997, A\&A, 317, L59

Galazutdinov, G. A., LoCurto, G., \& Krełowski, J. 2008, ApJ, 682, 1076

Gerin, M., de Luca, M., Black, J., et al. 2010, A\&A, 518, L110

Gredel, R. 1999, A\&A, 351, 657

Gredel, R., \& Muench, G. 1986, A\&A, 154, 336

Gredel, R., van Dishoeck, E. F., \& Black, J. H. 1991, A\&A, 251, 625

Gredel, R., van Dishoeck, E. F., \& Black, J. H. 1993, A\&A, 269, 477

Gredel, R., Pineau des Forêts, G., \& Federman, S. R. 2002, A\&A, 389, 993

Gummersbach, C. A., \& Kauffer, A. 1993, in The Hot Star Newsletter, 22, 16

Habart, E., Natta, A., \& Krügel, E. 2004, A\&A, 427, 179

Hendel, W., Khan, Z. H., \& Schmidt, W. 1986, Tetrahedron, 42, 1127

Henning, T., \& Salama, F. 1998, Science, 282, 220

Hobbs, L. M., York, D. G., Thorburn, J. A., et al. 2009, ApJ, 705, 32

Iglesias-Groth, S., Manchado, A., García-Hernández, D. A., Hernández, J. I. G., \& Lambert, D. L. 2008, ApJ, 685, L55

Iglesias-Groth, S., Manchado, A., Rebolo, R., et al. 2010, MNRAS, 407, 2157

Joblin, C., Léger, A., \& Martin, P. 1992, ApJ, 393, L79
Karcher, W., Fordham, R. J., Dubois, J. J., Glaude, P. G. J. M., \& Lighart, J. A. M. (eds.) 1985, Spectral Atlas of Polycyclic Aromatic Compounds, vol. 1 (Dordrecht: D. Reidel)

Kaźmierczak, M., Schmidt, M. R., Galazutdinov, G. A., et al. 2010, MNRAS, 408, 1590

Kerr, T. H., Hibbins, R. E., Miles, J. R., et al. 1996, MNRAS, 283, L105

Kokkin, D. L., Troy, T. P., Nakajima, M., et al. 2008, ApJ, 681, L49

Krełowski, J., Beletsky, Y., \& Galazutdinov, G. A. 2010a, ApJ, 719, L20

Krełowski, J., Beletsky, Y., Galazutdinov, G. A., et al. 2010b, ApJ, 714, L64

Kurucz, R. L. 1991, in NATO ASIC Proc. 341: Stellar Atmospheres - Beyond Classical Models, 441

Le Page, V., Snow, T. P., \& Bierbaum, V. M. 2003, ApJ, 584, 316

Leger, A., \& Puget, J. L. 1984, A\&A, 137, L5

Lepp, S., \& Dalgarno, A. 1988a, ApJ, 335, 769

Lepp, S., \& Dalgarno, A. 1988b, ApJ, 324, 553

Lepp, S., Dalgarno, A., van Dishoeck, E. F., \& Black, J. H. 1988, ApJ, 329, 418

Lien, D. J. 1984, ApJ, 284, 578

Linder, B., \& Abdulnur, S. 1971, J. Chem. Phys., 54, 1807

Livengood, T. A., Fast, K. E., Kostiuk, T., et al. 1999, PASP, 111, 512

London, F. 1937, Trans. Faraday Soc., 33, 8b

Longuet-Higgins, H. C., \& Pople, J. A. 1957, J. Chem. Phys., 27, 192

Maier, J. P., Walker, G. A. H., Bohlender, D. A., et al. 2011, ApJ, 726, 41

Malloci, G., Mulas, G., \& Joblin, C. 2004, A\&A, 426, 105

Malloci, G., Joblin, C., \& Mulas, G. 2007, Chem. Phys., 332, 353

Marcelino, N., Cernicharo, J., Agúndez, M., et al. 2007, ApJ, 665, L127

Mattila, K. 1986, A\&A, 160, 157

McCarthy, M. C., Gottlieb, C. A., Gupta, H., \& Thaddeus, P. 2006, ApJ, 652, L141

McCall, B. J., Thorburn, J., Hobbs, L. M., Oka, T., \& York, D. G. 2001, ApJ, 559, L49

Mennella, V., Colangeli, L., Bussoletti, E., Palumbo, P., \& Rotundi, A. 1998, ApJ, 507, L177

Meyer, D. M., \& Roth, K. C. 1991, ApJ, 376, L49

Meyer, D. M., Roth, K. C., \& Hawkins, I. 1989, ApJ, 343, L1

Millar, T. J., Walsh, C., Cordiner, M. A., Ní Chuimín, R., \& Herbst, E. 2007, ApJ, 662, L87

Motylewski, T., Linnartz, H., Vaizert, O., et al. 2000, ApJ, 531, 312

Mulliken, R. S. 1939, J. Chem. Phys., 7, 14

O'Keefe, A., \& Deacon, D. A. G. 1988, Rev. Sci. Instr., 59, 2544

Radzig, A. A., \& Smirnov, B. M. 1985, in Reference Data on Atoms, Molecules, and Ions, Springer Series in Chemical Physics 31, ed. J. P. Toennies (Heidelberg: Springer-Verlag)

Remijan, A. J., Hollis, J. M., Lovas, F. J., et al. 2007, ApJ, 664, L47

Romanini, D., Biennier, L., Salama, F., et al. 1999, Chem. Phys. Lett., 303, 165

Rouillé, G., Krasnokutski, S., Huisken, F., et al. 2004, J. Chem. Phys., 120, 6028

Rouillé, G., Arold, M., Staicu, A., et al. 2007, J. Chem. Phys., 126, 174311

Rouillé, G., Steglich, M., Huisken, F., Henning, T., \& Müllen, K. 2009, J. Chem. Phys., 131, 204311

Salama, F., Bakes, E. L. O., Allamandola, L. J., \& Tielens, A. G. G. M. 1996, ApJ, 458, 621

Salama, F., Galazutdinov, G. A., Krełowski, J., Allamandola, L. J., \& Musaev, F. A. 1999, ApJ, 526, 265

Shalev, E., Ben-Horin, N., Even, U., \& Jortner, J. 1991a, J. Chem. Phys., 95, 3147

Shalev, E., Ben-Horin, N., \& Jortner, J. 1991b, Chem. Phys. Lett., 177, 161

Shalev, E., Ben-Horin, N., \& Jortner, J. 1992, J. Chem. Phys., 96, 1848

Smith, A. L. 1996, J. Phys. B: At., Mol. Opt. Phys., 29, 4975

Sonnentrucker, P., Cami, J., Ehrenfreund, P., \& Foing, B. H. 1997, A\&A, 327, 1215

Staicu, A., Rouillé, G., Sukhorukov, O., Henning, T., \& Huisken, F. 2004, Mol. Phys., 102, 1777

Staicu, A., Rouillé, G., Henning, T., et al. 2008, J. Chem. Phys., 129, 074302

Steglich, M., Jäger, C., Rouillé, G., et al. 2010, ApJ, 712, L16

Sukhorukov, O., Staicu, A., Diegel, E., et al. 2004, Chem. Phys. Lett., 386, 259

Tan, X., \& Salama, F. 2005, J. Chem. Phys., 123, 014312

Tan, X., \& Salama, F. 2006, Chem. Phys. Lett., 422, 518

Thaddeus, P., Gottlieb, C. A., Gupta, H., et al. 2008, ApJ, 677, 1132

Tielens, A. G. G. M. 2008, ARA\&A, 46, 289

Tulej, M., Kirkwood, D. A., Pachkov, M., \& Maier, J. P. 1998, ApJ, 506, L69

Walsh, C., Harada, N., Herbst, E., \& Millar, T. J. 2009, ApJ, 700, 752

Welty, D. E., Federman, S. R., Gredel, R., Thorburn, J. A., \& Lambert, D. L. 2006, ApJS, 165, 138

Weselak, T., Galazutdinov, G. A., Beletsky, Y., \& Krełowski, J. 2009a, MNRAS, 400, 392

Weselak, T., Galazutdinov, G. A., Musaev, F. A., Beletsky, Y., \& Krełowski, J. 2009b, A\&A, 495, 189

Wyrowski, F., Menten, K. M., Güsten, R., \& Belloche, A. 2010, A\&A, 518, A26 